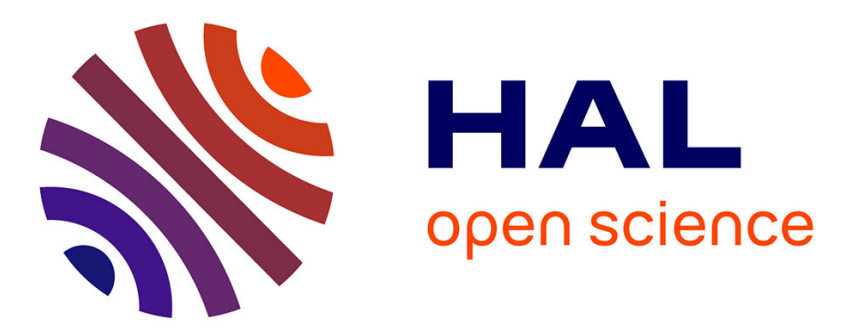

\title{
Three-dimensional extension of Lighthill's large-amplitude elongated-body theory of fish locomotion
}

Fabien Candelier, Frédéric Boyer, Alban Leroyer

\section{- To cite this version:}

Fabien Candelier, Frédéric Boyer, Alban Leroyer. Three-dimensional extension of Lighthill's largeamplitude elongated-body theory of fish locomotion. Journal of Fluid Mechanics, 2011, 674, pp.196226. 10.1017/S002211201000649X . hal-01145115

\author{
HAL Id: hal-01145115 \\ https://hal.science/hal-01145115
}

Submitted on 15 Nov 2018

HAL is a multi-disciplinary open access archive for the deposit and dissemination of scientific research documents, whether they are published or not. The documents may come from teaching and research institutions in France or abroad, or from public or private research centers.
L'archive ouverte pluridisciplinaire HAL, est destinée au dépôt et à la diffusion de documents scientifiques de niveau recherche, publiés ou non, émanant des établissements d'enseignement et de recherche français ou étrangers, des laboratoires publics ou privés. 


\title{
Three-dimensional extension of Lighthill's large-amplitude elongated-body theory of fish locomotion
}

\author{
Fabien Candelier ${ }^{1}$, Frédéric Boyer ${ }^{2}$, Alban Leroyer ${ }^{3}$ \\ ${ }^{1}$ Université Aix-Marseille 2, IUSTI, Technopôle de Château Gombert, 60 rue Joliot-Curie, \\ 13453 Marseille CEDEX 13, France \\ ${ }^{2}$ Ecole des Mines de Nantes, La Chantrerie, 4 rue Alfred Kastler, \\ BP 20722, 44307 Nantes, France \\ ${ }^{3}$ Laboratoire de Mécanique des Fluides, Ecole Centrale Nantes, 1 rue de la Noë, BP 92101, \\ 44321 Nantes CEDEX 3, France
}

\begin{abstract}
The goal of this paper is to derive expressions for the pressure forces and moments acting on an elongated body swimming in a quiescent fluid. The body is modelled as an inextensible and unshearable (Kirchhoff) beam, whose cross-sections are elliptic, undergoing prescribed deformations, consisting of yaw and pitch bending. The surrounding fluid is assumed to be inviscid, and irrotational everywhere, except in a thin vortical wake. The Laplace equation and the corresponding Neumann boundary conditions are first written in terms of the body coordinates of a beam treating the body as a fixed surface. They are then simplified according to the slenderness of the body and its kinematics. Because the equations are linear, the velocity potential is sought as a sum of two terms which are linked respectively to the axial movements of the beam and to its lateral movements. The lateral component of the velocity potential is decomposed further into two sub-components, in order to exhibit explicitly the role of the two-dimensional potential flow produced by the lateral motion of the cross-section, and the role played by the curvature effects of the beam on the crosssectional flow. The pressure, which is given by Bernoulli's equation, is integrated along the body surface, and the expressions for the resultant and the moment are derived analytically. Thereafter, the validity of the force and moment obtained analytically is checked by comparisons with Navier-Stokes simulations (using Reynolds-averaged Navier-Stokes equations), and relatively good agreements are observed.
\end{abstract}

Key words: swimming/flying

\section{Introduction}

The understanding of the mechanism of propulsion or, more precisely, the determination of the force and the moment experienced by a swimming slender fish has been a challenging topic for several decades. This problem has many fundamental aspects not only for fluid mechanicians but also for other scientific communities such as biologists or bio-inspired roboticians. As a result, the literature in this 
area is extensive and we shall not attempt a detailed review here. The interested reader can find numerous theoretical, experimental or numerical reviews that analyse the hydrodynamics of swimming fish such as Sfakiotakis, Lane \& Davies (1999), Triantafyllou, Triantafyllou \& Yue (2000), Sparenberg (2002), Colgate \& Lynch (2004) or Blake (2004).

At first sight, the modelling of undulatory fish motion looks rather complex, if not insoluble, due to major difficulties that must be overcome in order to develop a comprehensive theory. This probably explains why many researchers working on the topic have chosen to focus their attention on the wake generated by the swimming fish and, in particular, on the shed vorticity. This indeed is probably a promising way to provide, one day, a theory that combines both resistive (viscous) and reactive (pressure) effects. In particular, numerous studies tend to demonstrate that the high performance of swimming fish, in terms of efficiency and manoeuvrability, lies in their ability to control, through their body shape, the large-scale vortices shed in their wake (Wolfgang 1999; Triantafyllou et al. 2000; Zhu et al. 2002). Owing to the continuous improvement in computational fluid dynamics methods, these phenomena have recently been extensively investigated (Kern \& Koumoutsakos 2006; Eldredge 2008) which enables more accurate and complex simulations. As a necessary complement to these numerical approaches, experimental works using the particleimage velocimetry (PIV) method (Muller, Stamhuis \& Videler 2000) have emphasized the complex interactions of bound and tail vortices in the wake of swimming fish.

Nevertheless, when fast calculations are needed, such as when using the model for the on-line control of fish-like robots, full numerical approaches are still not well adapted as they are time-consuming. Therefore, finding accurate analytical expressions for the force and the moment acting on a moving and deformable body is still a challenging task for fluid mechanicians (see for instance the recent contributions of Kanso et al 2005; Kanso 2009; Munnier 2009). Historically, seminal contributions in the range of high Reynolds numbers were made by Wu (1961) and Lighthill (1960). The first, due to $\mathrm{Wu}$ (1961), is based on the undulating infinite height plate, while the second, due to Lighthill (1960), is an extension of the classical slender-body theory (see for instance Batchelor 1967, §6.9), called the elongated-body theory (EBT). In both cases, the fluid is inviscid and the modelling takes advantage of the characteristics of fish geometry in order to approximate the three-dimensional (3D) flow around the fish body by a stratification (horizontal in the first case and vertical in the second) of planar potential flows. In particular, in the EBT, it is considered that for each transverse slice of a fish, the lateral flow corresponds to the potential flow produced by the movement of an infinite cylinder of the same cross-section.

Since then, many improvements in the EBT have been proposed by several authors. For instance, Newman \& Wu (1973) and Newman (1973) took into account the effects of the body fin on the pressure resultant while Lighthill (1970) proposed an extension of the EBT, called the large-amplitude elongated-body theory (hereinafter LAEBT) in order to account for large amplitude motions.

This theory remains one of the basic results of biofluiddynamics, valuable for deriving estimates of the force acting on a swimming body (Weihs 1972, 1973 or Lighthill 1975) or fast simulation and control in robotics (Boyer et al. 2008).

However, in recent years, this theory has become a topic for discussion among fluid mechanicians. In particular, numerical studies have shown that in each transverse slice of a fish, the flow differs significantly from the potential flow produced by the movement of an infinite cylinder of the same cross-section (Wolfgang 1999; Triantafyllou et al. 2000). Therefore, the objectives of this paper are twofold: to 
(a)

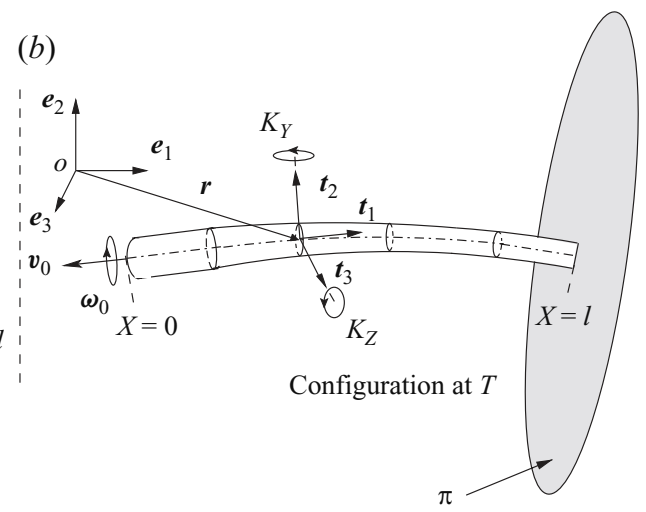

FIGURE 1. Sketch of the transformation.

provide a better understanding of the physical reality of the flow underlying the LAEBT and to generalize this theory to 3D swimming, including a possible rotation (roll) of the body around its vertebral axis.

To do this, we propose, following Lighthill (1960), to determine analytically (at leading order) the velocity potential of the fluid surrounding the body, and then, to calculate directly the resultant (and here the moment) of the pressure forces exerted by the flow at its surface. As in the LAEBT, the elongated body considered here is made up of elliptic transverse sections whose size may vary along the body, which has no appendices. Its 3D movements are provided by prescribing an internal pitch bending in addition to the yaw motion required by planar swimming. Note that to carry out this calculation, it is essential to change the coordinates so that the body becomes a fixed surface. Usually, when we are concerned with a deformable body moving in an inviscid fluid, the classical approach is to measure the deformation fields from a mobile (floating) frame that follows the rigid motion of the body, using Kirchhoff potentials method (Galper \& Miloh 1995, 1999). However, in the present investigation, we have chosen to make use of a different transformation, written in an inertial frame, which relies on a nonlinear beam theory introduced by Simo \& Vu-Locq (1988), and said to be geometrically exact. This transformation is presented in $\S 2$, and after that it is applied to the equations governing the flow around the body. Section 3 is devoted to the explicit calculation of the pressure resultant and moment exerted by the fluid on the body and the results are compared with numerical simulations in $\S 4$. Finally, in $\S 5$, the results are discussed.

\section{Transformation of coordinates}

\subsection{The body model}

The transformation of coordinates used in this investigation is inspired by those proposed by Reissner (1973) and Simo \& Vu-Locq (1988). Let $\mathscr{R}_{0}=\left(o, \boldsymbol{e}_{1}, \boldsymbol{e}_{2}, \boldsymbol{e}_{3}\right)$ be the laboratory reference frame. Without losing generality, it may be assumed that, at the initial time, the body experiences no deformations, as shown in figure $1(a)$, the idea being to introduce a transformation that links the current position of the body to its initial position. Note that in the following, vectors which refer to the initial position will be written upper case, while lower case is used for vectors which refer to the current position. Owing to the elongated geometry of the slender body, it can be considered as a Kirchhoff (Cosserat) beam $\mathscr{B}$, i.e. a one-dimensional 
continuum of rigid cross-sections (here elliptic) along a curve (here the backbone). Let $\mathscr{F}_{b}=\left(O, \boldsymbol{E}_{1}, \boldsymbol{E}_{2}, \boldsymbol{E}_{3}\right)$ be a Cartesian (body) frame attached to the reference (initial) configuration, in which the beam is assumed to be straight and aligned along $\left(O, \boldsymbol{E}_{1}\right)$. As we can see in figure $1(a), X \boldsymbol{E}_{1}$ is the position of any cross-section's centre $G(X, T=0)$, so that any rigid section can be labelled only by its axial coordinate $X$ and therefore denoted by $\mathscr{S}_{X}$.

Thereafter, when the body is moving, a continuous set of moving orthonormal frames $\mathscr{F}_{m}(X, T)=\left(G, \boldsymbol{t}_{1}, \boldsymbol{t}_{2}, \boldsymbol{t}_{3}\right)$ are attached to each cross-section, such that $\boldsymbol{t}_{1}(X, T)$ is the outward unit normal of the section as represented in figure 1(b). According to this parametrization, any material point, located at $\boldsymbol{x}$, belonging to the body during an admissible motion is related to its initial position by the following one-to-one mapping:

$$
\boldsymbol{x}=\boldsymbol{r}(X, T)+\boldsymbol{x}_{\perp},
$$

where $\boldsymbol{x}_{\perp}=\boldsymbol{R}(X, T) \cdot \boldsymbol{X}_{\perp}$. Here, $\boldsymbol{r}$ is the position vector of $G(X, t)$ in the laboratory frame (located initially at $\left.X \boldsymbol{E}_{1}\right), \boldsymbol{R}(X, t)$ is a rotation operator (i.e. $\boldsymbol{R}^{T}=\boldsymbol{R}^{-1}$ ) mapping $\left(\boldsymbol{E}_{1}, \boldsymbol{E}_{2}, \boldsymbol{E}_{3}\right)$ onto $\left(\boldsymbol{t}_{1}, \boldsymbol{t}_{2}, \boldsymbol{t}_{3}\right)$, while if $\boldsymbol{X}=X \boldsymbol{E}_{1}+Y \boldsymbol{E}_{1}+Z \boldsymbol{E}_{3}$ is the position vector of a material point in the reference configuration, then $\boldsymbol{X}_{\perp}=\boldsymbol{X}-\boldsymbol{X} \boldsymbol{E}_{1}$ and $\boldsymbol{x}_{\perp}$ correspond, respectively, to the position of the same point relative to the backbone in the reference and the deformed configuration. According to (2.1), the velocity field of any material point belonging to the body is entirely specified by two fields that depend only on $(X, T)$,

$$
\boldsymbol{v}=\frac{\partial \boldsymbol{r}}{\partial T}+\boldsymbol{\omega} \times \boldsymbol{x}_{\perp},
$$

the first field corresponding to the linear velocities of the mass centres of the rigid cross-sections (initially located at $X$ ),

$$
\frac{\partial \boldsymbol{r}}{\partial T}=V_{X} \boldsymbol{t}_{1}+V_{Y} \boldsymbol{t}_{2}+V_{Z} \boldsymbol{t}_{3},
$$

and the second to their angular velocities,

$$
\omega=\Omega_{X} t_{1}+\Omega_{Y} t_{2}+\Omega_{Z} t_{3} .
$$

Note that the angular velocity field is linked to the tensor $\boldsymbol{R}$ by the following relation:

$$
\boldsymbol{\omega} \times \boldsymbol{y}=\left(\frac{\partial \boldsymbol{R}}{\partial T} \cdot \boldsymbol{R}^{T}\right) \cdot \boldsymbol{y} \triangleq \hat{\boldsymbol{\omega}} \cdot \boldsymbol{y}, \quad \forall \boldsymbol{y},
$$

where the symbol $\triangleq$ stands for 'equal by definition'.

Similarly, in this approach, the strain state of the body is also totally specified by two vector fields, also depending only on $(X, T)$, which correspond to the tangent field of the material axis of the body $\mathscr{B}$ :

$$
\frac{\partial \boldsymbol{r}}{\partial X},
$$

and to the torsion and curvature vector field of the sections:

$$
\boldsymbol{k}=K_{X} \boldsymbol{t}_{1}+K_{Y} \boldsymbol{t}_{2}+K_{Z} \boldsymbol{t}_{3},
$$

which is also linked to $\boldsymbol{R}$ by the relation

$$
\boldsymbol{k} \times \boldsymbol{y}=\left(\frac{\partial \boldsymbol{R}}{\partial X} \cdot \boldsymbol{R}^{T}\right) \cdot \boldsymbol{y} \triangleq \hat{\boldsymbol{k}} \cdot \boldsymbol{y}, \quad \forall \boldsymbol{y} .
$$


The frame field $\left(G, t_{1}, t_{2}, t_{3}\right)$ defined by the transformation proposed by Simo (1988) is materially attached to the rigid cross-sections. In particular, the frame $\left(G, t_{1}, t_{2}, t_{3}\right)$ differs significantly from the more usual Frenet frame, which is defined only by the position of the centreline of the body since, in any circumstance, the local frames $\left(G, t_{1}, t_{2}, t_{3}\right)$ are always principal inertial frames of the cross-sections. Furthermore, the geometrically exact approach introduces a torsion field which is not a geometrical quantity defined by the centreline only, but an actual torsion field defined as a measure of the internal torsion strain state. Consequently, this material torsion field $\left(K_{X}\right)$ is never singular, even when the centreline tends to be a straight line, in contrast to the geometrical torsion field defined by the Frenet frame. Note that, physically, the other components of $\boldsymbol{k}$, that is $K_{Y}$ and $K_{Z}$, can be interpreted as the inverse of the radius of curvature of the body in the planes $\left(t_{1}, t_{3}\right)$ and $\left(t_{1}, t_{2}\right)$, respectively. If we now return to the swimming of a natural fish, the variables $K_{Y} \mathrm{~d} X$ and $K_{Z} \mathrm{~d} X$ are merely the two infinitesimal angles of rotation (standing for the pitch and yaw rotations along the backbone) of an infinitesimal Cardan joint, which links any pair of contiguous cross-sections together.

In what follows, $K_{Y}$ and $K_{Z}$ are considered as time-dependent control inputs, which entirely parametrize the body shapes. Since the elongated body of a vertebrate animal admits no relative rotation of two contiguous vertebrae about the vertebral axis, it is natural to impose that the torsion strain field satisfies

$$
K_{X}=0 .
$$

Strictly speaking, if we denote by $K$ the order of approximation of $K_{Y}$ and $K_{Z}$ and by $l$ the length of the body, the beam model presented here is valid only within the limit

$$
K l \lesssim O(1)
$$

which nevertheless allows large deformations to be considered, as those experienced by a swimming (manoeuvring) slender fish. In addition, it will be assumed that the body is inextensible and sufficiently slender to be considered as unshearable. Mathematically, these assumptions read

$$
\frac{\partial \boldsymbol{r}}{\partial X}=\boldsymbol{t}_{1} .
$$

According to (2.9) and (2.11), the body can be considered now as an untwistable and inextensible Kirchhoff beam, and the corresponding gradient transformation tensor reads

$$
\boldsymbol{F}(\boldsymbol{X})=\boldsymbol{R} \cdot \boldsymbol{U},
$$

where we recognize the polar decomposition which is valuable for any non-singular transformation with $\boldsymbol{U}$ being the tensor of pure deformation, defined as

$$
\boldsymbol{U}=\left(\begin{array}{ccc}
1+K_{Y} Z-K_{Z} Y & 0 & 0 \\
0 & 1 & 0 \\
0 & 0 & 1
\end{array}\right)
$$

In this context, the metric tensor of the transformation is given by

$$
\boldsymbol{G}=\boldsymbol{F}^{T} \cdot \boldsymbol{F}=\boldsymbol{U}^{T} \cdot \boldsymbol{U},
$$

and in the following, we will also be often concerned with the term $\sqrt{|\mathbf{G}|}$ (where $|\cdot|$ stands for the matrix determinant) which corresponds physically to a measure of local volume dilatations and contractions generated by the transformation. Also, 
during the investigation, this analysis will be in a domain restricted to the vicinity of the body, where $K_{Y} Z \sim K_{Z} Y \ll 1$, so that we can write

$$
\sqrt{|\mathbf{G}|}=1+K_{Y} Z-K_{Z} Y,
$$

since there is no ambiguity about the sign of this term. So far, we have described a transformation that links any current configuration of the body to its initial position. In what follows, it is shown that this transformation also provides relevant information about the kinematics of an inextensible and unshearable slender body.

\subsection{Kinematics of an inextensible and linear beam-like body}

When a slender body is moving, the orders of the velocities and the angular velocities are linked together, according to the assumptions of inextensibility and unshearability. Indeed, if we derive (2.11) with respect to $T$ and also use the fact that derivatives with respect to $X$ and to $T$ commute, it is simple to show that

$$
\frac{\partial}{\partial X}\left(\frac{\partial \boldsymbol{r}}{\partial T} \cdot \boldsymbol{t}_{i}\right)=\frac{\partial \boldsymbol{t}_{1}}{\partial T} \cdot \boldsymbol{t}_{i}+\frac{\partial \boldsymbol{r}}{\partial T} \cdot \frac{\partial \boldsymbol{t}_{i}}{\partial X} \quad \text { for } \quad i=\{1,2,3\} .
$$

In addition, since we also have

$$
\frac{\partial \boldsymbol{t}_{i}}{\partial T}=\boldsymbol{\omega} \times \boldsymbol{t}_{i} \quad \text { and } \quad \frac{\partial \boldsymbol{t}_{i}}{\partial X}=\boldsymbol{k} \times \boldsymbol{t}_{i},
$$

then we obtain the following three relations:

$$
\begin{aligned}
& \frac{\partial V_{X}}{\partial X}=K_{Z} V_{Y}-K_{Y} V_{Z}, \\
& \frac{\partial V_{Y}}{\partial X}=\Omega_{Z}-V_{X} K_{Z}, \\
& \frac{\partial V_{Z}}{\partial X}=-\Omega_{Y}+V_{X} K_{Y} .
\end{aligned}
$$

Similar relations can also be obtained for the angular velocities. Indeed, if we use $\partial\left(\boldsymbol{R}^{T} \cdot \boldsymbol{R}\right) / \partial T=0$, together with the definition $(2.5)$ of $\hat{\boldsymbol{\omega}}$, then it becomes possible to show that $\partial \boldsymbol{R}^{T} / \partial T=-\boldsymbol{R}^{T} \cdot \hat{\boldsymbol{\omega}}$, and subsequently, from the definition of $\hat{\boldsymbol{k}}$ (see (2.8)), it follows that

$$
\frac{\partial \hat{\boldsymbol{k}}}{\partial T}=\hat{\boldsymbol{\omega}} \cdot \hat{\boldsymbol{k}}-\hat{\boldsymbol{k}} \cdot \hat{\boldsymbol{\omega}}+\frac{\partial \hat{\boldsymbol{\omega}}}{\partial X}
$$

By writing (2.21) in terms of components and using (2.9), we obtain

$$
\begin{aligned}
\frac{\partial \Omega_{X}}{\partial X} & =K_{Z} \Omega_{Y}-K_{Y} \Omega_{Z} \\
\frac{\partial \Omega_{Y}}{\partial X} & =\frac{\partial K_{Y}}{\partial T}-K_{Z} \Omega_{X} \\
\frac{\partial \Omega_{Z}}{\partial X} & =\frac{\partial K_{Z}}{\partial T}+K_{Y} \Omega_{X} .
\end{aligned}
$$

As we can see, (2.18)-(2.20) and (2.22)-(2.24) provide us with ideas about the relative order of the derivatives with respect to $X$ of the components of the linear and angular velocities of an inextensible and unshearable slender body. In particular, if we denote by $V$ the order of the different components of the translational velocity (i.e. $V \sim V_{X} \sim V_{Y} \sim V_{Z}$ ) and by $\Omega_{\perp}$ the order of $\Omega_{Y}$ and $\Omega_{Z}$, then, according to (2.19), 
(2.20) and (2.10), we see that

$$
\frac{\alpha \Omega_{\perp}}{V} \lesssim O(\varepsilon), \quad \text { where } \quad \varepsilon=\frac{\alpha}{l} \ll 1,
$$

and $\alpha$ stands for the typical length scale of the cross-sections. This result will be used in what follows.

\subsection{Velocity potential equations in terms of the body coordinates}

In this problem, the surrounding fluid is assumed to be inviscid, and the flow to be irrotational everywhere except in a thin vortical wake which occurs downstream of the caudal fin and takes the form of a vortex sheet (at least just behind the trailing edge). Note that in theory, it should be expected that this vortex sheet produces an additional flow which can be determined by the Biot-Savart law. In particular, such effects are taken into account when the inviscid fluid equations are solved with the boundary element method (with the Kutta condition) (see Wolfgang 1999). In the present investigation however, and in common with the original theory of Lighthill (EBT), the pressure perturbation due to the wake-induced flow is expected to be negligible, and as a consequence, once it is shed in the flow, the vorticity is later ignored. In this condition, it is possible to limit the analytical investigation within a domain located upstream of a flat surface $\pi$ perpendicular to the caudal fin, without specifying particular boundary conditions for the potential in this plane. Thus, if $\phi$ denotes the velocity potential in this domain, its governing equations read, in a reference frame in which the fluid far from the body is at rest, as

$$
\begin{gathered}
\Delta \phi(\boldsymbol{y}, t)=0, \\
\nabla \phi \cdot \boldsymbol{n}=\boldsymbol{v} \cdot \boldsymbol{n}, \quad \text { on } \partial \mathscr{B}, \\
\nabla \phi \rightarrow \mathbf{0}, \quad|\boldsymbol{y}-\boldsymbol{x}| \rightarrow \infty .
\end{gathered}
$$

Then, if the body transformation of coordinates presented in the previous section is applied to (2.26) and (2.27), we obtain

$$
\frac{\partial}{\partial X}\left(\frac{1}{\sqrt{|\boldsymbol{G}|}} \frac{\partial \Phi}{\partial X}\right)+\frac{\partial}{\partial Y}\left(\sqrt{|\boldsymbol{G}|} \frac{\partial \Phi}{\partial Y}\right)+\frac{\partial}{\partial Z}\left(\sqrt{|\boldsymbol{G}|} \frac{\partial \Phi}{\partial Z}\right)=0
$$

and

$$
\begin{aligned}
\frac{1}{|\mathbf{G}|} \frac{\partial \Phi}{\partial X} N_{X}+\frac{\partial \Phi}{\partial Y} N_{Y}+\frac{\partial \Phi}{\partial Z} N_{Z}= & \frac{1}{\sqrt{|\mathbf{G}|}}\left(V_{X}+\Omega_{Y} Z-\Omega_{Z} Y\right) N_{X} \\
& +\left(V_{Y}-\Omega_{X} Z\right) N_{Y}+\left(V_{Z}+\Omega_{X} Y\right) N_{Z}
\end{aligned}
$$

(Note that we have not written the transformation of (2.28) since it is not used explicitly in the following.)

In order to analyse the order of the different terms occurring in (2.29) and (2.30), we denote by $N_{\|}$the order of $N_{X}$ and by $N_{\perp}$ the order of $N_{Y}$ or $N_{Z}$. Owing to the slenderness of the body, we first have necessarily

$$
N_{\|} \sim \varepsilon N_{\perp} .
$$

Furthermore, since the typical length scale for $Y$ and $Z$ is given by $\alpha$ (at least in the vicinity of the body) and because

$$
K \alpha \sim K l \varepsilon
$$


we have

$$
\sqrt{|\mathbf{G}|} \sim 1+K l \varepsilon
$$

In contrast with $Y$ and $Z$, the typical length of the axial coordinates $X$ is given by $l$. As a result, in such a problem, variations of the variables with respect to $X$ are weak compared with those within the cross-sectional plane. (Roughly, it may be considered that $\partial / \partial X \sim 1 / l$, whereas $\partial / \partial Y \sim \partial / \partial Z \sim 1 / \alpha)$. If we exploit this, as well as the geometrical relations given by (2.31) and (2.33) and the kinematic relation given by (2.25), then (2.29) and (2.30) can be simplified as follows:

$$
\begin{gathered}
\frac{\partial^{2} \Phi}{\partial Y^{2}}+\frac{\partial^{2} \Phi}{\partial Z^{2}} \simeq K_{Z} \frac{\partial \Phi}{\partial Y}-K_{Y} \frac{\partial \Phi}{\partial Z} \\
\frac{\partial \Phi}{\partial Y} N_{Y}+\frac{\partial \Phi}{\partial Z} N_{Z} \simeq V_{X} N_{X}+\left(V_{Y}-\Omega_{X} Z\right) N_{Y}+\left(V_{Z}+\Omega_{X} Y\right) N_{Z},
\end{gathered}
$$

where the terms that scale as $o\left(\varepsilon^{2}\right)$ compared with leading order terms have been neglected. (Note that, for simplicity, 'small' terms scale as $o\left(\varepsilon^{2}\right)$ compared with leading order.)

\subsubsection{Decomposition of the velocity potential}

If we now use the linearity of (2.34) and (2.35), the solution of these equations may be sought in the form

$$
\Phi=\Phi_{\|}+\Phi_{\perp}
$$

where $\Phi_{\|}$and $\Phi_{\perp}$ are solutions respectively of

$$
\begin{gathered}
\frac{\partial^{2} \Phi_{\|}}{\partial Y^{2}}+\frac{\partial^{2} \Phi_{\|}}{\partial Z^{2}} \simeq 0, \\
\frac{\partial \Phi_{\|}}{\partial Y} N_{Y}+\frac{\partial \Phi_{\|}}{\partial Z} N_{Z} \simeq V_{X} N_{X},
\end{gathered}
$$

and

$$
\begin{gathered}
\frac{\partial^{2} \Phi_{\perp}}{\partial Y^{2}}+\frac{\partial^{2} \Phi_{\perp}}{\partial Z^{2}} \simeq K_{Z} \frac{\partial \Phi_{\perp}}{\partial Y}-K_{Y} \frac{\partial \Phi_{\perp}}{\partial Z} \\
\frac{\partial \Phi_{\perp}}{\partial Y} N_{Y}+\frac{\partial \Phi_{\perp}}{\partial Z} N_{Z} \simeq\left(V_{Y}-\Omega_{X} Z\right) N_{Y}+\left(V_{Z}+\Omega_{X} Y\right) N_{Z}
\end{gathered}
$$

Note that the right-hand-side terms of (2.37) can be neglected, since due to $N_{\|} \sim \varepsilon N_{\perp}$, $\Phi_{\|}$scales like $\varepsilon \alpha V$. In this context, we also observe that the potential $\Phi_{\|}$may be interpreted as a pseudo slender-body-like potential, where the velocity $V_{X}$ varies along $X$ (in contrast with what happens when we deal with a classical rigid slender body), whereas $\Phi_{\perp}$ accounts for the lateral motion and axial (roll) rotation of the body when it is swimming.

With now the lateral component $\Phi_{\perp}$, a serious issue occurs, since the solution of (2.39) is not easily achieved because of the non-zero right-hand side. Fortunately, this difficulty can be circumvented if we use a perturbation method to determine $\Phi_{\perp}$, at least at the order we are interested in. In the present investigation, according to (2.32), the solution of (2.39) and (2.40) can indeed be sought in the form

$$
\Phi_{\perp} \simeq \Phi_{\perp}^{0}+\Phi_{\perp}^{1}
$$

where

$$
\frac{\Phi_{\perp}^{1}}{\Phi_{\perp}^{0}} \sim O(\alpha K)
$$


and $\Phi_{\perp}^{0}$ is the solution of (2.39)-(2.40), when $K_{Y}=K_{Z}=0$, i e. $\Phi_{\perp}^{0}$ is the solution of

$$
\frac{\partial^{2} \Phi_{\perp}^{0}}{\partial Y^{2}}+\frac{\partial^{2} \Phi_{\perp}^{0}}{\partial Z^{2}}=0
$$

with the boundary conditions

$$
\frac{\partial \Phi_{\perp}^{0}}{\partial Y} N_{Y}+\frac{\partial \Phi_{\perp}^{0}}{\partial Z} N_{Z}=\left(V_{Y}-\Omega_{X} Z\right) N_{Y}+\left(V_{Z}+\Omega_{X} Y\right) N_{Z}
$$

Therefore, if we inject (2.41) into (2.39) and (2.40) and if the small terms are once again neglected, then the equations governing $\Phi_{\perp}^{1} \mathrm{read}$

$$
\frac{\partial^{2} \Phi_{\perp}^{1}}{\partial Y^{2}}+\frac{\partial^{2} \Phi_{\perp}^{1}}{\partial Z^{2}}=K_{Z} \frac{\partial \Phi_{\perp}^{0}}{\partial Y}-K_{Y} \frac{\partial \Phi_{\perp}^{0}}{\partial Z}
$$

and

$$
\frac{\partial \Phi_{\perp}^{1}}{\partial Y} N_{Y}+\frac{\partial \Phi_{\perp}^{1}}{\partial Z} N_{Z}=0
$$

Note that the explicit analytical expressions of $\Phi_{\perp}^{0}$ and $\Phi_{\perp}^{1}$ are needed in the following to determine the pressure force and the moment acting on the body when it is swimming. Their derivations are detailed in the Appendix. Instead, in what follows, we illustrate the flow produced by each part of the velocity potential in typical situations encountered in planar swimming.

\subsection{Cross-sectional flow features}

During the planar swimming of a slender fish, the propulsion is ensured by a wave of deformation which travels the body, and whose amplitude increases gradually from head to tail. Near the head, the curvature is often negligible and the lateral velocities of the cross-sections are generally small compared with the axial velocity. As a consequence, the lateral flow that is observed in such a cross-section is almost only generated by $\Phi_{\|}$. Figure 2 shows the flow produced by a pure axial motion of the slender body, in a normalized case where the axial velocity has been set equal to -1 , and where the dimensions of the elliptic cross-section of the body, whose major and minor semi-axes denoted respectively $\alpha$ and $\beta$ below, have been set equal to 1 and 0.5 , and where $\partial \alpha / \partial X=\partial \beta / \partial X=0.1$. When the body moves axially, it pushes the surrounding fluid laterally, as illustrated by the directions of the streamlines. Note that the pattern of the streamlines can be qualitatively compared with reasonably good agreement with the numerical results presented by Triantafyllou et al. (2000).

In contrast, near the tail, the local curvature of the body may become significant. If it is assumed that the cross-sectional area is almost constant with respect to $X$ (i.e. $N_{X}=0$ ), then the flow that takes place in a cross-section is, at this time, only produced by $\Phi_{\perp}$. Figure 3 shows such a flow, again in a normalized case, where the lateral velocity $V_{Z}$ has been set equal to 1 and the normalized curvature reads $K_{Y}=0.25$. As shown previously, this cross-sectional flow results from the sum of a two-dimensional (2D) potential flow $\left(\Phi_{\perp}^{0}\right)$ around an infinite cylinder, which is displayed in figure 4, and a perturbation flow produced by $\Phi_{\perp}^{1}$, which is due to curvature effects of the body on the flow, and is displayed in figure 5 .

In order to better understand the physical origin of this perturbation flow, it is worth recalling that by definition, the flow produced by the potential $\Phi_{\perp}^{0}$ is in a direction perpendicular to the backbone. Let us therefore consider an infinitesimal volume of control, surrounding the body surface and lying between the abscissa $X$ and $X+\mathrm{d} X$ and whose lateral sections remain perpendicular to the backbone 


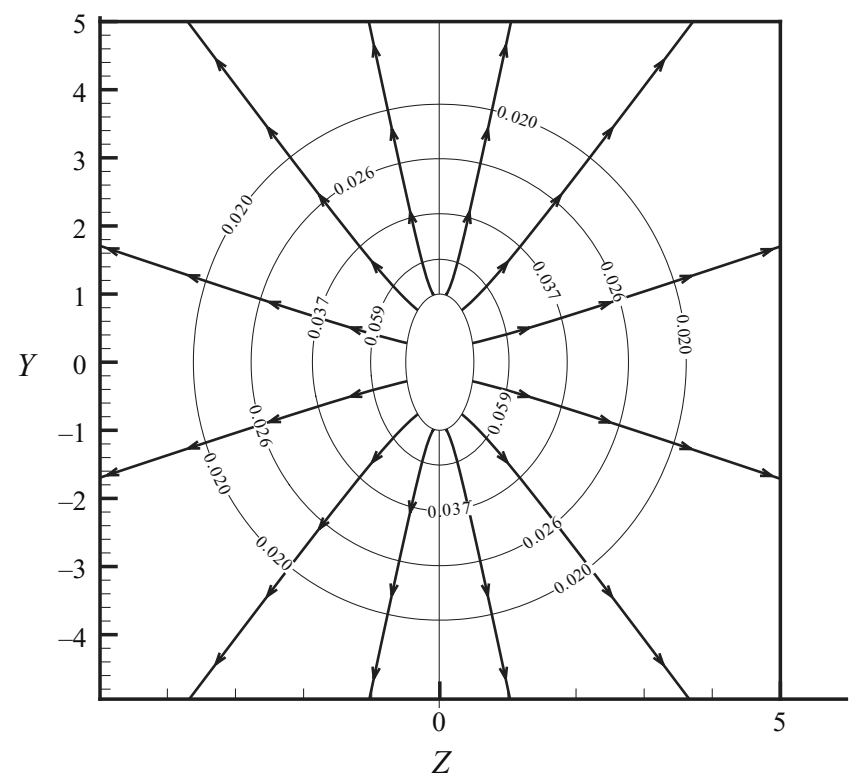

FIGURE 2. Slender-body flow produced by $\Phi_{\|}\left(V_{X}=-1, V_{Z}=0, K_{Y}=0\right)$.

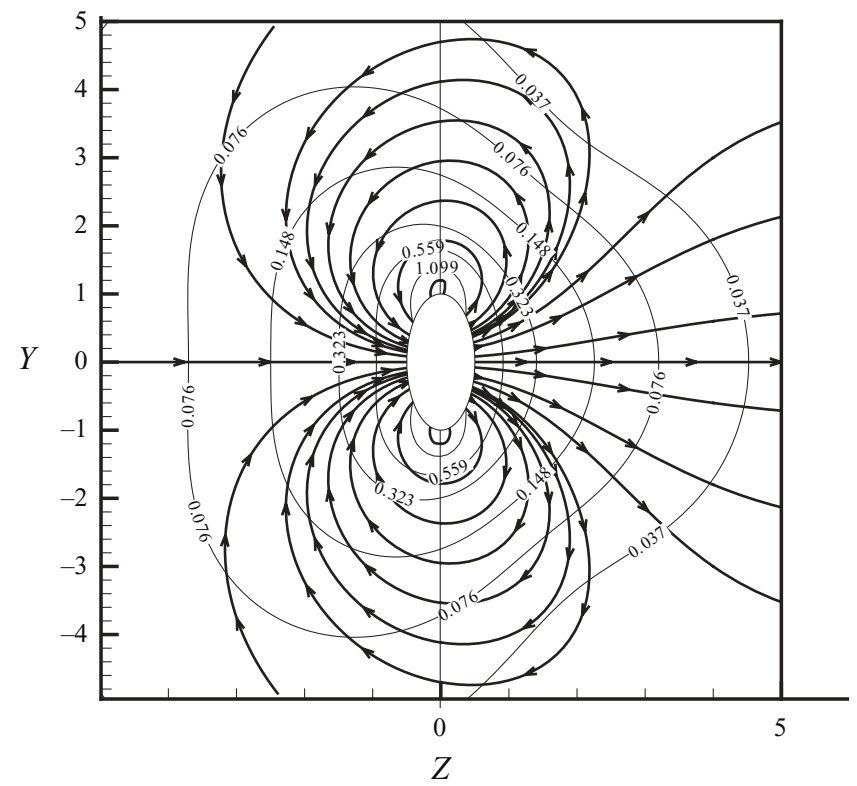

FigURE 3. Lateral flow in a cross-section produced by $\Phi_{\perp}\left(V_{Z}=1, K_{Y}=0.25\right)$.

during the deformation of the body, as illustrated in figure 6. In this example, $V_{Z}>0$ and, since $K_{Y}>0$, the surface of the volume corresponding to the entering fluid is smaller than the surface corresponding to the outgoing fluid. Hence, according to the continuity equation, the outgoing fluid velocity must be smaller than the entering fluid velocity. Because the velocity produced by $\Phi_{\perp}^{0}$ is symmetrical, we see therefore that an additional velocity (which is encoded in $\Phi_{\perp}^{1}$ ) must take place within 


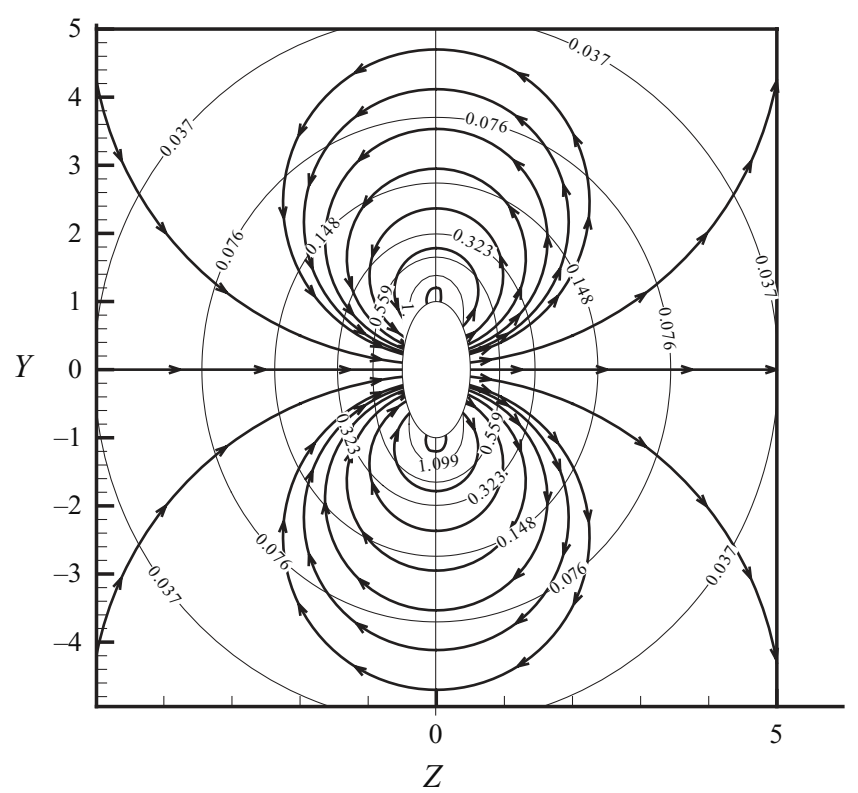

Figure 4. Two-dimensional potential flow $\left(N_{X}=0, V_{Z}=1\right)$.

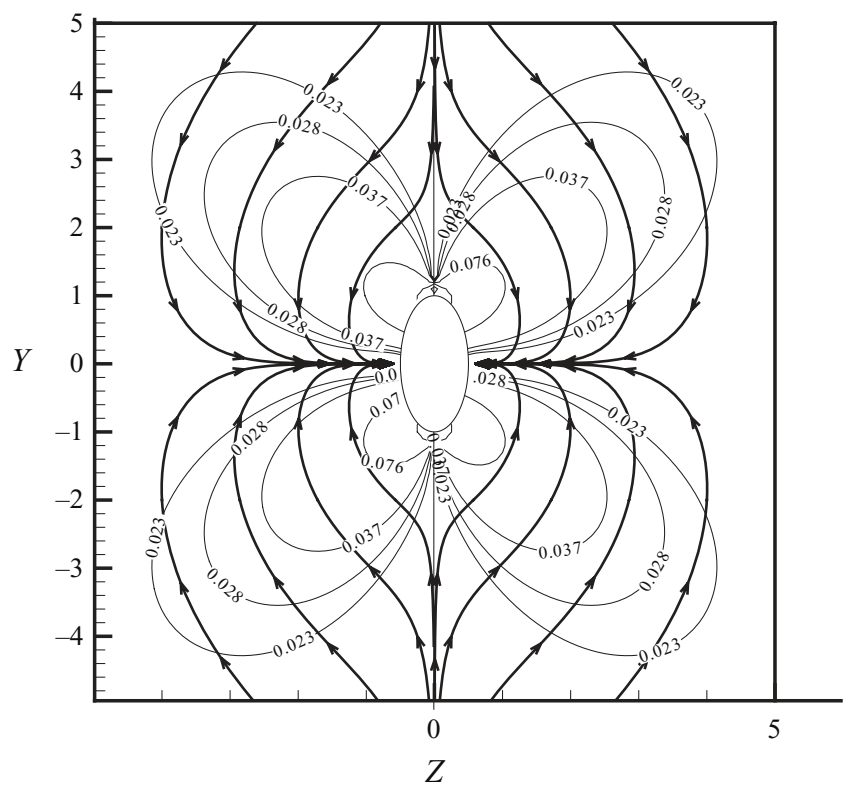

Figure 5. Flow produced by $\Phi_{\perp}^{1}\left(N_{X}=0, V_{Z}=1, K_{Y}=0.25\right)$.

the cross-section, in order to satisfy the continuity equation. Also, we see that the cross-sectional flow underlying the LAEBT is actually more rich than the 2D potential flow produced by the movement of an infinite cylinder of the same cross-section involved in the EBT. Note that the $2 \mathrm{D}$ potential flow and the perturbation flow are both symmetrical, but the former is symmetrical in relation to the axis $\left(G, t_{3}\right)$ whereas the latter is only symmetrical in relation to the axis $\left(G, t_{2}\right)$, and as a result, their 


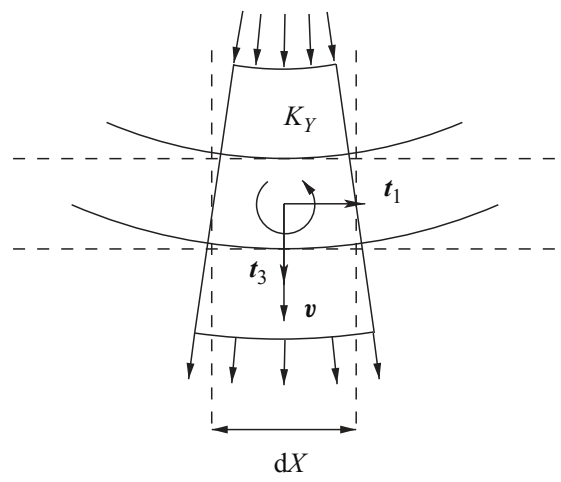

FIGURE 6. Illustration of the body deformation effects on the flow.

sum is no longer symmetrical. Again, the typical patterns of the streamlines can be qualitatively compared, with relatively good agreement, with the numerical results presented by Triantafyllou et al. (2000).

So far, we have discussed the flow generated by the velocity potential $\Phi$. In what follows, it is shown that this potential is also consistent with the existence of a vortical wake occurring downstream of the caudal fin of the body.

\subsection{Vortex shedding}

First of all, let us recall that to formulate the LAEBT, Lighthill (1975) considered the rate of change of momentum within a volume of fluid enclosing the fish, whose boundary at each instant includes a flat surface $\pi$ (see figure 1 ). In this approach, one of the main difficulties he had to overcome was to determine the resultant of the pressures generated by the fluid motion within this caudal plane. As noted by Lighthill (1970), a possible interpretation of this resultant rests on the existence of a vortical wake which occurs downstream of the caudal fin. This idea was later investigated by Childress (1981), who recovered the pressure resultant within the caudal plane by considering the energy balance in a volume of control downstream of the caudal fin that involved the wake energy. Childress (1981, p. 104) also provided an analytical approach which gets around the Kutta condition by using Kelvin's circulation theorem to estimate the vorticity shed at the trailing edge. Following his statements, if we denote as $\delta \Phi(Y)$, the difference of the potential on either side of the trailing edge, and at the same height of the cross-section (i.e. $Y$ ), then when the body moves forward, the two components of the shed vorticity read

$$
\gamma_{Y}=-\frac{1}{V_{X}(l)} \frac{\partial \delta \Phi}{\partial T} \quad \text { and } \quad \gamma_{X}=-\frac{\partial \delta \Phi}{\partial Y},
$$

where $V_{X}(l)$ corresponds to the axial velocity of the caudal fin (see figure 7). From a practical point of view, a sharp trailing edge is nothing but an elliptic crosssection whose minor semi-axis (i.e. $\beta$ ) tends to zero, and therefore, in terms of elliptic coordinates (see the Appendix), the difference of the potential simply reads

$$
\delta \Phi=\Phi\left(u_{X}=0, v\right)-\Phi\left(u_{X}=0,2 \pi-v\right) .
$$

According to the analytical solutions of $\Phi$ (see Appendix), together with when $u_{X} \rightarrow 0$, the Cartesian coordinate $Y$ is linked to the angular elliptic coordinate by the relation 


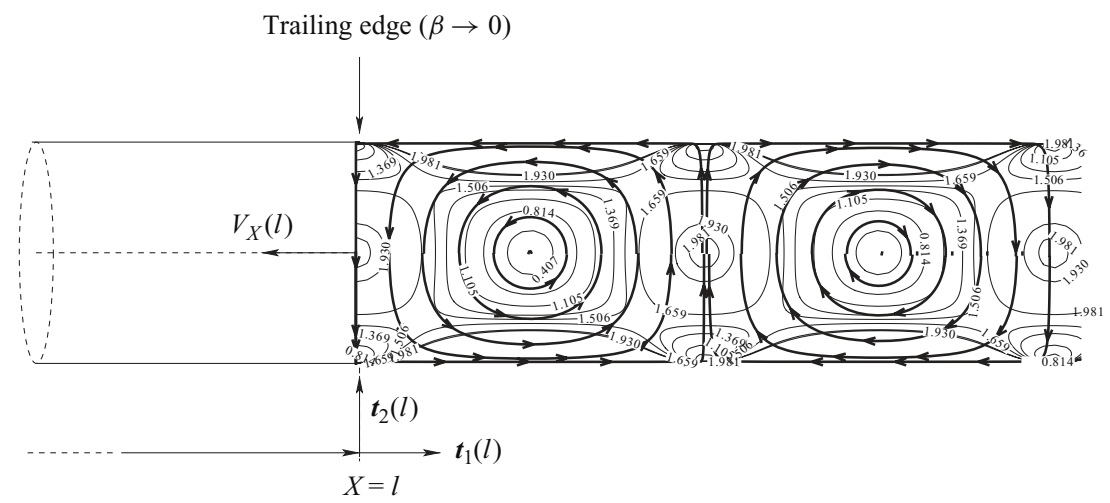

FIGURE 7. Illustration of the shed vorticity during planar swimming.

$Y=\alpha \cos (v)$, and the difference of the velocity potential involved in this investigation can be written in the form

$$
\delta \Phi=\sqrt{\alpha^{2}-Y^{2}}\left(-2 V_{Z}+\frac{\alpha^{2} \Omega_{X} K_{Z}}{6}-\left(\Omega_{X}+\frac{V_{Z} K_{Z}}{2}\right) Y-\frac{\Omega_{X} K_{Z}}{6} Y^{2}\right) .
$$

Strictly speaking, this analytical approach provides only a model of the shed vorticity, and the actual vortical wake is probably extremely complex. Nevertheless, in order to further illustrate the flow underlying the LAEBT theory, let us assume that immediately downstream of the trailing edge, the wake is defined by a material surface of frozen vortex lines, forming a vortical sheet. Then, by extending the curvilinear body coordinates $(X, Y)$ into the wake, the vorticity located at a distance $X$ from the trailing edge is linked to the time $T$ taken by the caudal fin to travel this distance by the following relation:

$$
X(T)-l=\int_{0}^{T}-V_{X}(l) \mathrm{d} \tau .
$$

According to (2.49), if it is also assumed that the axial velocity $V_{X}(l)$ is constant and the lateral velocity $V_{Z}(l)$ of the trailing edge is known as a time-dependent function, it is possible to reconstruct the vorticity field in the wake. Figure 7 illustrates such a field in a normalized case, corresponding to planar swimming and where we have set arbitrarily $V_{X}(l)=-1$ and $V_{Z}(l)=\sin (T)$. Note that, in reality, once shed into the wake, such a sheet of vorticity is actually highly unstable and splits into vortex rings (see for instance the numerical simulations presented by Kern \& Koumoutsakos 2006).

\section{Force and moment acting on the body}

In terms of the body coordinates $X, Y$ and $Z$, it can be shown that the force and the moment acting on the beam are given by the following integrals:

$$
\boldsymbol{f}_{h}=\int_{\partial \mathscr{B}}-P \sqrt{|\boldsymbol{G}|} \boldsymbol{F}^{-T} \cdot \boldsymbol{N} \mathrm{d} S \quad \text { and } \quad \boldsymbol{m}_{h}=\int_{0}^{l} \boldsymbol{r} \times \frac{\partial \boldsymbol{f}_{h}}{\partial X} \mathrm{~d} X+\boldsymbol{c}_{h},
$$


where $c_{h}$ corresponds to the integration of the density of the hydrodynamic couple applied to the beam per unit of length

$$
\boldsymbol{c}_{h}=\int_{\partial \mathscr{B}}-P \boldsymbol{X}_{\perp} \times\left(\sqrt{|\mathbf{G}|} \boldsymbol{F}^{-T} \cdot \boldsymbol{N}\right) \mathrm{d} S,
$$

and where the pressure reads

$$
\begin{aligned}
P(\boldsymbol{X}, T)-P_{\infty}=-\rho\left(\frac{\partial \Phi}{\partial T}-\left(\boldsymbol { U } ^ { - 1 } \cdot \boldsymbol { R } ^ { T } \cdot \left(\frac{\partial \boldsymbol{r}}{\partial T}\right.\right.\right. & \left.\left.\left.+\omega \times \boldsymbol{x}_{\perp}\right)\right) \cdot \nabla_{X} \Phi\right) \\
& -\frac{\rho}{2}\left(\nabla_{X} \Phi\right) \cdot\left(\mathbf{G}^{-1} \cdot \nabla_{X} \Phi\right)
\end{aligned}
$$

In (3.3), if small terms are neglected, the pressure can be reorganized as follows:

$$
\begin{aligned}
P & -P_{\infty} \\
& \simeq \rho\left[-\frac{\partial \Phi_{\perp}^{0}}{\partial T}+\left(V_{Y}-\Omega_{X} Z\right) \frac{\partial \Phi_{\perp}^{0}}{\partial Y}+\left(V_{Z}+\Omega_{X} Y\right) \frac{\partial \Phi_{\perp}^{0}}{\partial Z}-\frac{1}{2}\left(\frac{\partial \Phi_{\perp}^{0}}{\partial Y}\right)^{2}-\frac{1}{2}\left(\frac{\partial \Phi_{\perp}^{0}}{\partial Z}\right)^{2}\right] \\
& +\rho\left[-\frac{\partial \Phi_{\|}^{0}}{\partial T}+V_{X} \frac{\partial \Phi_{\perp}^{0}}{\partial X}+\left(V_{Y}-\Omega_{X} Z-\frac{\partial \Phi_{\perp}^{0}}{\partial Y}\right) \frac{\partial \Phi_{\|}}{\partial Y}+\left(V_{Z}+\Omega_{X} Y-\frac{\partial \Phi_{\perp}^{0}}{\partial Z}\right) \frac{\partial \Phi_{\|}}{\partial Z}\right] \\
& +\rho\left[-\frac{\partial \Phi_{\perp}^{1}}{\partial T}+\left(V_{Y}-\Omega_{X} Z-\frac{\partial \Phi_{\perp}^{0}}{\partial Y}\right) \frac{\partial \Phi_{\perp}^{1}}{\partial Y}+\left(V_{Z}+\Omega_{X} Y-\frac{\partial \Phi_{\perp}^{0}}{\partial Z}\right) \frac{\partial \Phi_{\perp}^{1}}{\partial Z}\right],
\end{aligned}
$$

which we write as $P-P_{\infty}=P_{0}+P_{1}+P_{2}$. Note that, physically, $P_{0}$ corresponds to the unsteady pressure distribution that would act on a moving infinite cylinder of the same cross-section, and clearly, this part is the leading-order part of the pressure acting on the body. Here $P_{1}$ is the pressure correction brought by the coupling between the potentials $\Phi_{\|}$(linked to the axial motion of the body) and $\Phi_{\perp}^{0}$, whereas $P_{2}$ is another correction which corresponds to the coupling between the potentials $\Phi_{\perp}^{1}$ (encoding the curvature effects of the body on the fluid) and $\Phi_{\perp}^{0}$. In practice, it can be shown that

$$
\frac{P_{1}}{P_{0}}=O(\varepsilon) \quad \text { and } \quad \frac{P_{2}}{P_{0}}=O(K l \varepsilon)
$$

\subsection{The pressure resultant}

We now return to the first integral given in (3.1). By using (2.12) and (2.13) together with $\boldsymbol{t}_{i}=\boldsymbol{R} \cdot \boldsymbol{E}_{i}$, it is possible to show that

$$
\sqrt{|\mathbf{G}|} \boldsymbol{F}^{-T} \cdot \boldsymbol{N}=N_{X} \boldsymbol{t}_{1}+\sqrt{|\mathbf{G}|} N_{Y} \boldsymbol{t}_{2}+\sqrt{|\mathbf{G}|} N_{Z} \boldsymbol{t}_{3}
$$

and, since $N_{Y} \mathrm{~d} S=\mathrm{d} Z \mathrm{~d} X$ and $N_{Z} \mathrm{~d} S=-\mathrm{d} Y \mathrm{~d} X$, the force acting on the body is given by

$$
\boldsymbol{f}_{h}=\int_{\partial \mathscr{B}}-P \boldsymbol{t}_{1} N_{X} \mathrm{~d} S+\int_{0}^{l}\left\{\oint_{\mathscr{C}_{X}}-P \sqrt{|\mathbf{G}|} \boldsymbol{t}_{2} \mathrm{~d} Z-\oint_{\mathscr{C}_{X}}-P \sqrt{|\mathbf{G}|} \boldsymbol{t}_{3} \mathrm{~d} Y\right\} \mathrm{d} X,
$$

where $\mathscr{C}_{X}$ stands for the contour of the cross-section located at $X$. In the present investigation, if we remember that $N_{\|} \sim \varepsilon N_{\perp}$, and according to (3.5), (3.7) can be simplified by neglecting the integrals involving the terms $P_{1} N_{X}$ and $P_{2} N_{X}$, since they are not consistent with our level of approximation. Thus, if we consider only the 


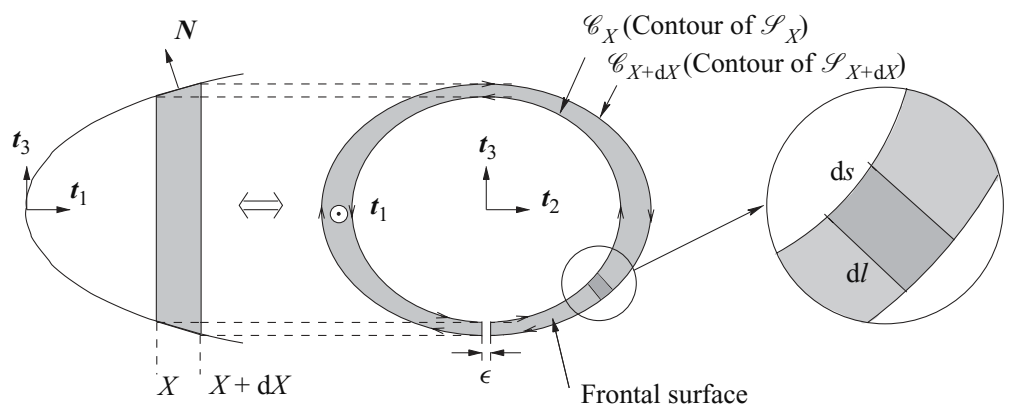

Figure 8. Integration surface.

terms that bring a non-negligible contribution, we obtain

$$
\begin{aligned}
\boldsymbol{f}_{h}= & \int_{\partial \mathscr{B}}-P_{0} \boldsymbol{t}_{1} N_{X} \mathrm{~d} \mathscr{S}+\int_{0}^{l}\left\{\oint_{\mathscr{C}_{X}}-P_{0} \boldsymbol{t}_{2} \mathrm{~d} Z-\oint_{\mathscr{C}_{X}}-P_{0} \boldsymbol{t}_{3} \mathrm{~d} Y\right\} \mathrm{d} X \\
& +\int_{0}^{l}\left\{\oint_{\mathscr{C}_{X}}-P_{1} \boldsymbol{t}_{2} \mathrm{~d} Z-\oint_{\mathscr{C}_{X}}-P_{1} \boldsymbol{t}_{3} \mathrm{~d} Y\right\} \mathrm{d} X \\
& +\int_{0}^{l}\left\{\oint_{\mathscr{C}_{X}}-P_{2} \boldsymbol{t}_{2} \mathrm{~d} Z-\oint_{\mathscr{C}_{X}}-P_{2} \boldsymbol{t}_{3} \mathrm{~d} Y\right\} \mathrm{d} X \\
& +\int_{0}^{l}\left\{\oint_{\mathscr{C}_{X}}-P_{0}\left(K_{Y} Z-K_{Z} Y\right) \mathrm{d} Z \boldsymbol{t}_{2}-\oint_{\mathscr{C}_{X}}-P_{0}\left(K_{Y} Z-K_{Z} Y\right) \mathrm{d} Y \boldsymbol{t}_{3}\right\} \mathrm{d} X,
\end{aligned}
$$

which we write as $\boldsymbol{f}_{h}=\boldsymbol{f}_{h 1}+\boldsymbol{f}_{h 2}+\boldsymbol{f}_{h 3}+\boldsymbol{f}_{h 4}+\boldsymbol{f}_{h 5}$. Actually, the first integral of (3.8) can be rewritten in such a manner that global force can also be written in the form

$$
\boldsymbol{f}_{h}=\int_{0}^{l} \sum_{i=1}^{5} \frac{\partial \boldsymbol{f}_{h i}}{\partial X} \mathrm{~d} X,
$$

where the integrand corresponds to the local cross-sectional loading acting on any body surface lying between the sections located at $X$ and $X+\mathrm{d} X$ (see figure 8). Physically, it may be interesting to emphasize the following.

(i) Here $\partial f_{h 1} / \partial X$ is the only axial part of the force density. This term occurs only if the section varies with respect to $X$. Indeed, in this case, the axial component $N_{X}$ is not null, and since it is coupled with $P_{0}$ (leading order), we obtain a small term of first order that must be taken into account.

(ii) Note that $\partial \boldsymbol{f}_{h 2} / \partial X$ is the lateral force density corresponding to the unsteady motion of an infinite cylinder of the same cross-section.

(iii) Also, $\partial \boldsymbol{f}_{h 3} / \partial X$ is the force density produced by the coupling between $\Phi_{\|}$and $\Phi_{\perp}^{0}$, that is between the axial motion of a slender-like body whose cross-section varies along $X$ and the lateral motion of the body. Note that such a coupling results from the nonlinearity of the Bernoulli equation.

(iv) In addition, $\partial \boldsymbol{f}_{h 4} / \partial X$ is the force density produced by the coupling between the lateral 2D potential $\Phi_{\perp}^{0}$ and the perturbation potential $\Phi_{\perp}^{1}$. This term is a consequence of the curvature effects on the surrounding flow.

(v) Note also that $\partial \boldsymbol{f}_{h 5} / \partial X$ is the force density produced by the stretching of the body surface which is exposed to the pressure $P_{0}$. This term is also a consequence of the curvature effects, but this time on the body. 
For the sake of clarity, the detailed derivations of these five components are not given here, and the resulting expression will be written directly. For an elliptic crosssection (whose major and minor semi-axes are $\alpha$ and $\beta$, respectively) the added-mass coefficients read (Milne-Thomson 1968)

$$
M_{Y}=\rho \pi \beta^{2}, \quad M_{Z}=\rho \pi \alpha^{2}, \quad M_{X}=\rho \pi \frac{\left(\alpha^{2}-\beta^{2}\right)^{2}}{8},
$$

respectively, and these coefficients are also involved in the density of linear fluid momentum expression

$$
\boldsymbol{p}_{f}=P_{Y} \boldsymbol{t}_{2}+P_{Z} \boldsymbol{t}_{3}
$$

where $P_{Y}=M_{Y} V_{Y}$ and $P_{Z}=M_{Z} V_{Z}$. According to these notations, the five force densities described previously read

$$
\begin{gathered}
\frac{\partial \boldsymbol{f}_{h 1}}{\partial X}=-\frac{1}{2}\left(\frac{\mathrm{d} M_{Y}}{\mathrm{~d} X} V_{Y}^{2}+\frac{\mathrm{d} M_{Y}}{\mathrm{~d} X} V_{Z}^{2}+\frac{\mathrm{d} M_{X}}{\mathrm{~d} X} \Omega_{X}^{2}\right) \boldsymbol{t}_{1}, \\
\frac{\partial \boldsymbol{f}_{h 2}}{\partial X}=-\left(\frac{\partial P_{Y}}{\partial T} \boldsymbol{t}_{2}+\frac{\partial P_{Z}}{\partial T} \boldsymbol{t}_{3}\right)-\Omega_{X} \boldsymbol{t}_{1} \times \boldsymbol{p}_{f}, \\
\frac{\partial \boldsymbol{f}_{h 3}}{\partial X}=V_{X}\left(\frac{\partial P_{Y}}{\partial X} \boldsymbol{t}_{2}+\frac{\partial P_{Z}}{\partial X} \boldsymbol{t}_{3}\right), \\
\frac{\partial \boldsymbol{f}_{h 4}}{\partial X} \simeq+\frac{1}{2} \frac{\beta M_{X}}{\alpha+\beta} \frac{\partial K_{Y} \Omega_{X} \boldsymbol{t}_{2}}{\partial T}+\frac{1}{2} \frac{\alpha M_{X}}{\alpha+\beta} \frac{\partial K_{Z} \Omega_{X} \boldsymbol{t}_{3}}{\partial T}+T_{f} \frac{\partial \boldsymbol{t}_{1}}{\partial X}
\end{gathered}
$$

and

$$
\frac{\partial \boldsymbol{f}_{h 5}}{\partial X}=-\frac{\beta^{2} M_{X}}{\alpha^{2}-\beta^{2}} \frac{\partial K_{Y} \Omega_{X} \boldsymbol{t}_{2}}{\partial T}+\frac{\alpha^{2} M_{X}}{\alpha^{2}-\beta^{2}} \frac{\partial K_{Z} \Omega_{X} \boldsymbol{t}_{3}}{\partial T}+\left(\frac{\partial V_{X}}{\partial X}\right) \boldsymbol{p}_{f}-2 T_{f} \frac{\partial \boldsymbol{t}_{1}}{\partial X} .
$$

We now focus on the combinations that are possible between these five equations, in order to simplify the final expression of the force. First, by summing $f_{h 2}$ and $f_{h 3}$, we obtain

$$
\boldsymbol{f}_{h 2}+\boldsymbol{f}_{h 3}=\int_{0}^{l}\left[-\left(\frac{\partial P_{Y}}{\partial T}-V_{X} \frac{\partial P_{Y}}{\partial X}\right) \boldsymbol{t}_{2}-\left(\frac{\partial P_{Z}}{\partial T}-V_{X} \frac{\partial P_{Z}}{\partial X}\right) \boldsymbol{t}_{3}-\Omega_{X} \boldsymbol{t}_{1} \times \boldsymbol{p}_{f}\right] \mathrm{d} X .
$$

Moreover, by using (2.17), (2.19) and (2.20), it is possible to show that

$$
\frac{\partial \boldsymbol{t}_{2}}{\partial T}-V_{X} \frac{\partial \boldsymbol{t}_{2}}{\partial X}=-\frac{\partial V_{Y}}{\partial X} \boldsymbol{t}_{1}+\Omega_{X} \boldsymbol{t}_{1} \times \boldsymbol{t}_{2},
$$

and

and therefore, it follows that

$$
\frac{\partial \boldsymbol{t}_{3}}{\partial T}-V_{X} \frac{\partial \boldsymbol{t}_{3}}{\partial X}=-\frac{\partial V_{Z}}{\partial X} \boldsymbol{t}_{1}+\Omega_{X} \boldsymbol{t}_{1} \times \boldsymbol{t}_{3},
$$

$$
\boldsymbol{f}_{h 2}+\boldsymbol{f}_{h 3}=\int_{0}^{l}\left[-\left(\frac{\partial \boldsymbol{p}_{f}}{\partial T}-V_{X} \frac{\partial \boldsymbol{p}_{f}}{\partial X}\right)-\left(P_{Y} \frac{\partial V_{Y}}{\partial X}+P_{Z} \frac{\partial V_{Z}}{\partial X}\right) \boldsymbol{t}_{1}\right] \mathrm{d} X .
$$

Now, if we sum $\boldsymbol{f}_{h 1}$ and $\boldsymbol{f}_{h 2}+\boldsymbol{f}_{h 3}$ and note that

$$
-\left(P_{Y} \frac{\partial V_{Y}}{\partial X}+P_{Z} \frac{\partial V_{Z}}{\partial X}\right)-\frac{1}{2}\left(\frac{\mathrm{d} M_{Y}}{\mathrm{~d} X} V_{Y}^{2}+\frac{\mathrm{d} M_{Z}}{\mathrm{~d} X} V_{Z}^{2}+\frac{\mathrm{d} M_{X}}{\mathrm{~d} X} \Omega_{X}^{2}\right) \simeq-\frac{\partial T_{f}}{\partial X},
$$


since the term $M_{X} \Omega_{X} \partial \Omega_{X} / \partial X$ is negligible, as we can see by (2.22), then we obtain

$$
\boldsymbol{f}_{h 1}+\boldsymbol{f}_{h 2}+\boldsymbol{f}_{h 3} \simeq \int_{0}^{l}-\left(\frac{\partial \boldsymbol{p}_{f}}{\partial T}-V_{X} \frac{\partial \boldsymbol{p}_{f}}{\partial X}\right)-\frac{\partial T_{f}}{\partial X} \boldsymbol{t}_{1} \mathrm{~d} X .
$$

This partial result actually corresponds to the force that would be obtained if we had considered only the 2D potential flow $\Phi_{\perp}^{0}$ (and its coupling with $\Phi_{\|}$), which results from the movement of an infinite cylinder whose cross-section is $\mathscr{S}_{X}$, without taking into account the effect of the curvature on the cross-sectional flow (given by $\Phi_{\perp}^{1}$ ) nor the deformation of the body surface. In other words, (3.22) corresponds to a 3D extension of the EBT proposed by Lighthill (1960). This result therefore provides us with the pressure force acting on the body when the deformations of the swimming body are small, that is, when $K l \ll O(1)$.

As to the part of the force coming from curvature effects, which must be taken into account when the deformations of the body are no longer negligible (i.e. $K l \sim O(1)$ ), it is given by

$$
\boldsymbol{f}_{h 4}+\boldsymbol{f}_{h 5}=\int_{0}^{l}\left(\frac{\partial V_{X}}{\partial X}\right) \boldsymbol{p}_{f}-T_{f} \frac{\partial \boldsymbol{t}_{1}}{\partial X}+M_{X Y} \frac{\partial K_{Y} \Omega_{X} \boldsymbol{t}_{2}}{\partial T}+M_{X Z} \frac{\partial K_{Z} \Omega_{X} \boldsymbol{t}_{3}}{\partial T} \mathrm{~d} X,
$$

where we have introduced, for simplicity, the coefficients

$$
M_{X Y}=\rho \frac{\pi \beta\left(\alpha^{2}-\beta^{2}\right)(\alpha-3 \beta)}{16} \text { and } \quad M_{X Z}=\rho \frac{\pi \alpha\left(\alpha^{2}-\beta^{2}\right)(3 \alpha-\beta)}{16} .
$$

Now, if we combine (3.22) and (3.23), we finally obtain the expression

$$
\boldsymbol{f}_{h}=\int_{0}^{l}-\frac{\partial \boldsymbol{p}_{f}}{\partial T}+\frac{\partial V_{X} \boldsymbol{p}_{f}}{\partial X}-\frac{\partial T_{f} \boldsymbol{t}_{1}}{\partial X} \mathrm{~d} X+\int_{0}^{l} M_{X Y} \frac{\partial K_{Y} \Omega_{X} \boldsymbol{t}_{2}}{\partial T}+M_{X Z} \frac{\partial K_{Z} \Omega_{X} \boldsymbol{t}_{3}}{\partial T} \mathrm{~d} X
$$

where it turns out that the part of the force due to curvature effects, that is (3.23), brings exactly what is necessary to introduce $V_{X}$ and $\boldsymbol{t}_{1}$ into the derivatives with respect to $X$ in the different terms of (3.22). As we can see, therefore, the first integral of (3.25) rigorously corresponds to the 3D extension of the LAEBT of Lighthill (1970), whereas the second integral accounts for a possible coupling between the roll and the body curvature.

Note that, for clarity, the main steps of the derivation of the final result are summarized in figure 9.

\subsection{The moment of hydrodynamic forces acting on the body}

So far, we have been interested in the pressure resultant but in order to determine totally the dynamics of the body (in particular for 3D swimming), it is also necessary to calculate the moment of forces acting on it, which is given by the second part of (3.1). As before, detailed derivations will not be given here, in order to simplify the reading. Let us simply note that if we use a very similar approach as for the resultant, 


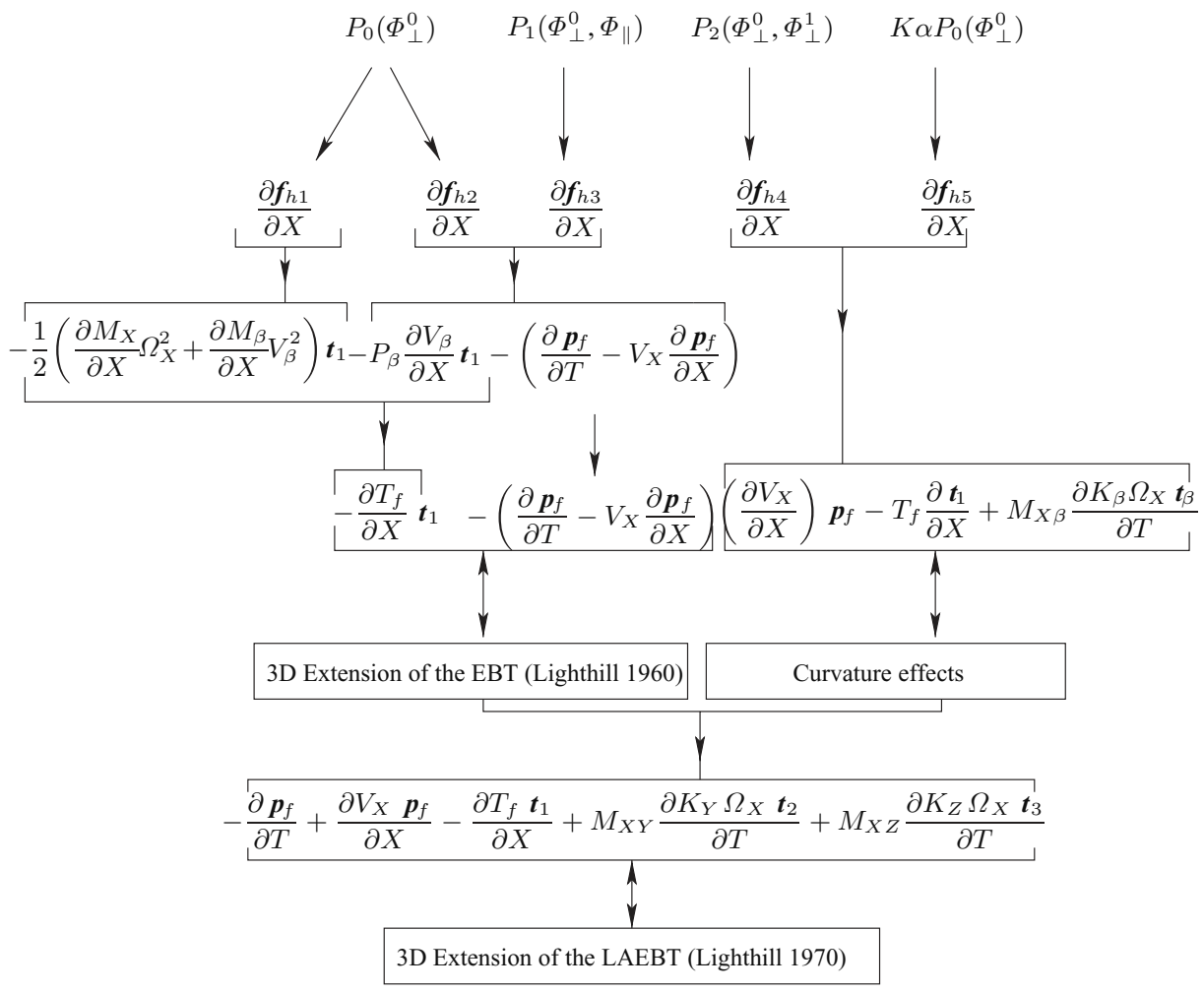

FIGURE 9. Diagram summarizing the main steps of the calculations leading to result (3.25).

then it can be shown that the moment acting on the body reads

$$
\begin{aligned}
\boldsymbol{m}_{h}= & -\frac{\partial}{\partial T} \int_{0}^{l}\left(\boldsymbol{\sigma}_{f}+\boldsymbol{r} \times \boldsymbol{p}_{f}\right) \mathrm{d} X+\left[V_{X}\left(\boldsymbol{\sigma}_{f}+\boldsymbol{r} \times \boldsymbol{p}_{f}\right)-\boldsymbol{r} \times T_{f} \boldsymbol{t}_{1}\right]_{0}^{l} \\
& +\frac{\partial}{\partial T} \int_{0}^{l}\left(\left(M_{X Y} K_{Y} V_{Y}+M_{X Z} K_{Z} V_{Z}\right) \boldsymbol{t}_{1}+\boldsymbol{r} \times\left(M_{X Y} K_{Y} \Omega_{X} \boldsymbol{t}_{2}+M_{X Z} K_{Z} \Omega_{X} \boldsymbol{t}_{3}\right)\right) \mathrm{d} X \\
& +\left[V_{Y} M_{X Y} \Omega_{X} \boldsymbol{t}_{2}+V_{Z} M_{X Z} \Omega_{X} \boldsymbol{t}_{3}\right]_{0}^{l}+\frac{\partial}{\partial T} \int_{0}^{l}\left(\tilde{M}_{Z} V_{Z} \boldsymbol{t}_{2}-\tilde{M}_{Y} V_{Y} \boldsymbol{t}_{3}\right) \mathrm{d} X \\
& +\int_{0}^{l}\left(\left(M_{X}-M_{X Y}\right) \frac{\partial V_{Y}}{\partial X} \Omega_{X} \boldsymbol{t}_{2}+\left(M_{X}-M_{X Z}\right) \frac{\partial V_{Z}}{\partial X} \Omega_{X} \boldsymbol{t}_{3}\right) \mathrm{d} X
\end{aligned}
$$

where $\sigma_{f}=M_{X} \Omega_{X} t_{1}$ represents the density of the roll kinetic momentum per unit of body length, $\boldsymbol{r}$ is the field of position on the backbone in relation to a reference point $o$ (see figure 1), and where we have introduced the new coefficients

$$
\tilde{M}_{Y}=\frac{\pi}{4} \alpha \beta\left(\alpha \frac{\partial \beta}{\partial X}+3 \beta \frac{\partial \alpha}{\partial X}\right) \quad \text { and } \quad \tilde{M}_{Z}=\frac{\pi}{4} \alpha \beta\left(\beta \frac{\partial \alpha}{\partial X}+3 \alpha \frac{\partial \beta}{\partial X}\right),
$$

which scale like $O(\varepsilon)$ compared with the classical added-mass coefficients. Note that concerning the moment, the physical origin of the different terms could be also identified as has been done previously for the cross-sectional forces, but it turns out that its expression does not benefit from the same fortunate arrangements, and therefore, we will not discuss this point further. 


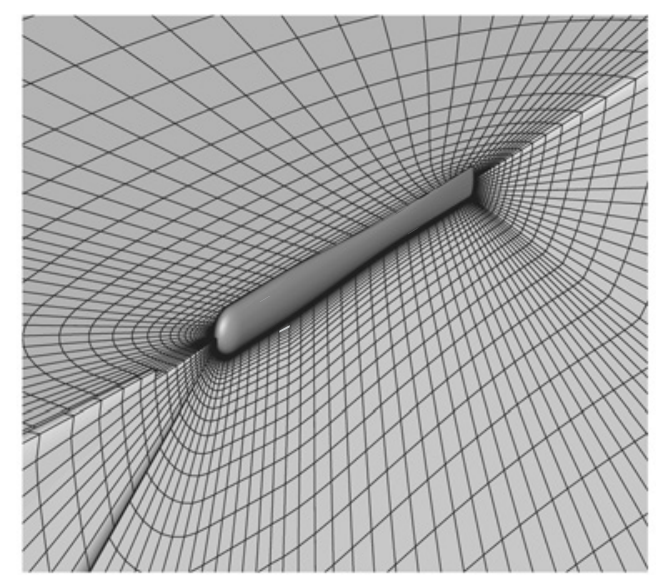

Figure 10. View of the body and the mesh around it.

\section{Comparisons with numerical results}

\subsection{Description of the numerical solver}

In order to check the validity of the fluid force model obtained previously, simulations using an incompressible unsteady Reynolds-averaged Navier-Stokes (RANS) solver are carried out. The in-house solver ISIS-CFD, developed at the Laboratory of Fluid Mechanics at Ecole Centrale Nantes, has been used for this task. The interested reader can find details of the numerical methodology in Leroyer \& Visonneau (2005). In brief, this approach performs numerical simulation of the RANS equations which are integrated by means of a second-order-accurate finite volume method. The velocity field is obtained from the momentum conservation equations and the pressure field is extracted from the mass conservation constraint or continuity equation, which is transformed into a pressure equation. Also, in the case of turbulent flows, additional transport equations for modelled variables are solved in a form similar to the momentum equations. Finally, in order to deal with moving bodies, an arbitrary Lagrangian-Eulerian (ALE) approach is used. It is associated with grid deformation techniques to keep the mesh fitted to the body, as its shape varies. The positioning of the body in space is then obtained using a rigid motion of this deformed mesh.

The mesh here is composed of 120000 cells spread in a block-structured grid (see figures 10 and 11).

\subsection{General settings: shape of the body and physical parameters}

Figure 10 shows the body geometry used in these simulations. It is composed of elliptical cross-sections obtained with the following process. The shape is first defined as a cylinder of diameter $D=0.1 l$ for any $X \in[0.05 l, 0.99 l]$, ended with two halfellipsoids between $X=[0,0.05 l]$ and $X=[0.99 l, l]$. Then, for any cross-section, the major axis (along $\left.E_{2}\right)$ is multiplied by $\kappa_{2}(X)$, and its minor axis (along $E_{3}$ ) by $\kappa_{3}(X)$, where $\kappa_{2}$ and $\kappa_{3}$ are two functions defined by

$$
\begin{aligned}
& \kappa_{2}(X)=1, \quad \text { for } 0 \leqslant X<0.2 l, \\
& \kappa_{2}(X)=1-\frac{\sin (\pi \tilde{X}-\pi / 2)+1}{5}, \text { for } 0.2 l \leqslant X<0.75 l, \quad \text { with } \tilde{X}=\frac{X-0.2 l}{0.75 l-0.2 l},
\end{aligned}
$$




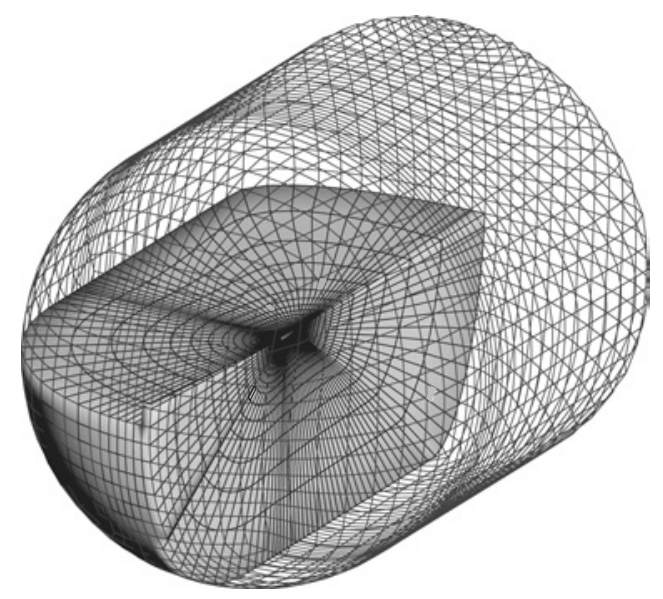

FIGURE 11. Global view of the fluid domain.

$$
\begin{aligned}
& \kappa_{2}(X)=\frac{3}{5}, \quad \text { for } 0.75 l \leqslant X<1, \\
& \kappa_{3}(X)=-\frac{1}{6}\left[\sin \left(\pi X-\frac{\pi}{2}\right)+1\right]-\frac{X^{2}}{8}+\frac{1}{2}, \quad \text { for } 0 \leqslant X<l .
\end{aligned}
$$

Here, the total length $l$ of the fish is set to $1 \mathrm{~m}$. As far as the fluid is concerned, the density and the dynamic viscosity are respectively set to $1000 \mathrm{~kg} \mathrm{~m}^{-3}$ and $0.001 \mathrm{~Pa} \mathrm{~s}$, similar to the properties of water.

\subsection{Numerical experimentations and comparisons with theoretical results}

As mentioned previously, the goal of these numerical simulations is to compare the body forces and moments given by (3.25) and (3.26) with those given by the Navier-Stokes simulation (using RANS equations). These comparisons are carried out by prescribing given motions of the body and by observing the resulting forces and moments. More precisely, in the cases considered, the body is simply towed by the head, and deforms according to imposed curvature laws, the complexity of which increases in time, in order to bring into play all the degrees of freedom of the body dynamics, and consequently, all the components of the models. During these numerical investigations, two different motions are considered, which follow the same protocol.

(i) In the first step, the body is towed straight forward by the head at a constant axial velocity $V_{0}=-0.6 \mathrm{~m} \mathrm{~s}^{-1}$ along the $X$-axis (leading to a Reynolds number equal to $6 \times 10^{5}$ ), and a planar deformation is imposed through the following curvature law:

$$
\begin{aligned}
K_{Y}=A \exp \left(\frac{s X}{l}-s\right)\left[\left(\left(\frac{s}{l}\right)^{2}-\left(\frac{2 \pi}{\lambda}\right)^{2}\right)\right. & \sin \left(\frac{2 \pi X}{\lambda}-\omega_{0} T\right) \\
& \left.+\frac{4 \pi s}{l \lambda} \cos \left(\frac{2 \pi X}{\lambda}-\omega_{0} T\right)\right],
\end{aligned}
$$

which is progressively applied (the establishing time is equal to one period of undulation, i.e. $\left.2 \pi / \omega_{0}\right)$ and thereafter maintained throughout the numerical run. Note that (4.5) was adapted from the work of Tytell (2004), who proposed a law of deflection for the backbone deduced from the experimental analysis of real eels, but here it has been expressed in terms of curvature.

(ii) After $2 \mathrm{~s}$, an unsteady rotation around the $X$-axis

$$
\Omega_{0}=\theta_{0} \omega_{0} \cos \left(\omega_{0} T\right)
$$


(a)

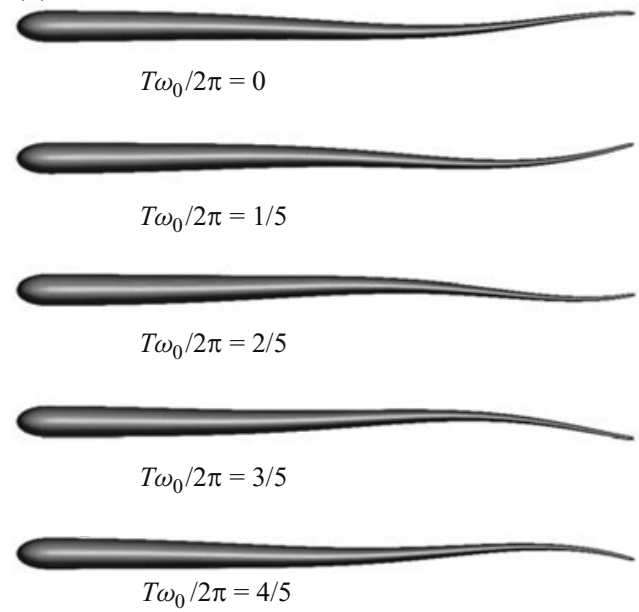

(b)
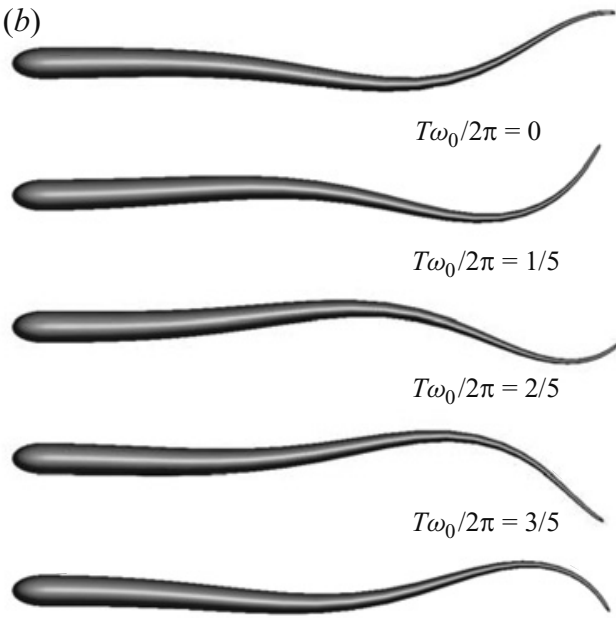

$T \omega_{0} / 2 \pi=4 / 5$

Figure 12. Deformations involved in $(a)$ motion 1 and $(b)$ motion 2.

is progressively imposed (again during one period of undulation), and this unsteady rotating motion is maintained until the end of the run. Note that $\theta_{0}$ is the angle corresponding to the rotation amplitude, and it has been arbitrarily set equal to $\pi / 6$.

In the deformation law given by (4.5), four parameters are involved, namely the amplitude of tail deflection $A$, a shape parameter $s$, the wave length $\lambda$ and the angular frequency $\omega_{0}$. In all runs, we have set $\omega_{0}=4 \pi \mathrm{s}^{-1}, \lambda=0.75 \mathrm{~m}$ and $s=4$, and the two motions tested differ by the value of the amplitude $A$. For motion $1, A=0.03 \mathrm{~m}$ whereas for motion $2, A=0.12 \mathrm{~m}$.

The typical deformations corresponding to these two prescribed motions are presented in figures $12(a)$ and $12(b)$. As we can see, deformations involved in the first motion are quite limited, so that they remain more or less inside the frame of the EBT, whereas deformations involved in the second case are more important. This second motion will enable us to check more specifically the validity of the LAEBT.

The ISIS-CFD computation starts at time $T=0$ with a converged solution of the flow for the body rigidly towed at the forward speed equal to $-V_{0} \boldsymbol{e}_{1}$, and the pressure forces and moments acting on the body are calculated in the head frame of the body, so that in the following, the components $F_{X}, F_{Y}$ and $F_{Z}$ are given in the basis $\left(t_{1}(0, T), t_{2}(0, T), t_{3}(0, T)\right)$, and moments $M_{X}, M_{Y}$ and $M_{Z}$ are defined in the same basis and related to the head of the body (i.e. the point $X=0$ ) .

Figures $13(a-c)$ and $13(d-f)$ show the fluid pressure forces acting on the body obtained numerically and those obtained with the analytical model, respectively for the two motions, whereas figures $14(a-c)$ and $14(d-f)$ compare the moments. Three dash-dotted vertical lines are also plotted in these graphs, delimiting respectively the end of the transient phase where the amplitude increases, the start of the rotation and the end of its transient phase.

We first discuss the results related to the fluid force directed along the head axis $t_{1}(0, T)$, i.e. $F_{X}$ obtained in response to the two motions. In figure 13(a) (it is also the case for figure $13 d$, but the range prevents its observation), we observe a small discrepancy at time $T=0$ between the CFD simulation and the model. This is probably due to the rounded-nose geometry of the body (see figure 10), since the pressure drag acting on the head along the $X$-axis is not captured by the 

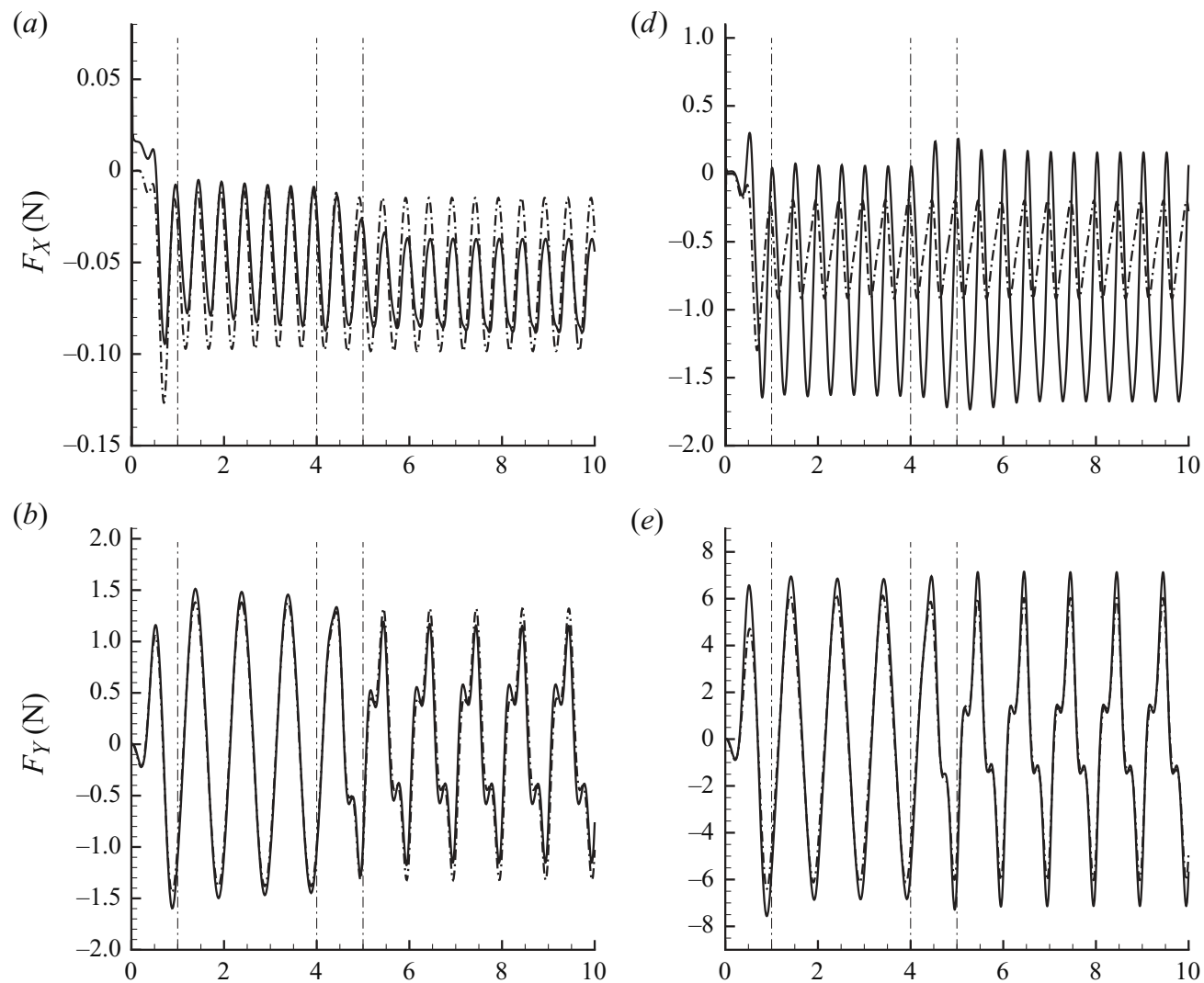

(e)
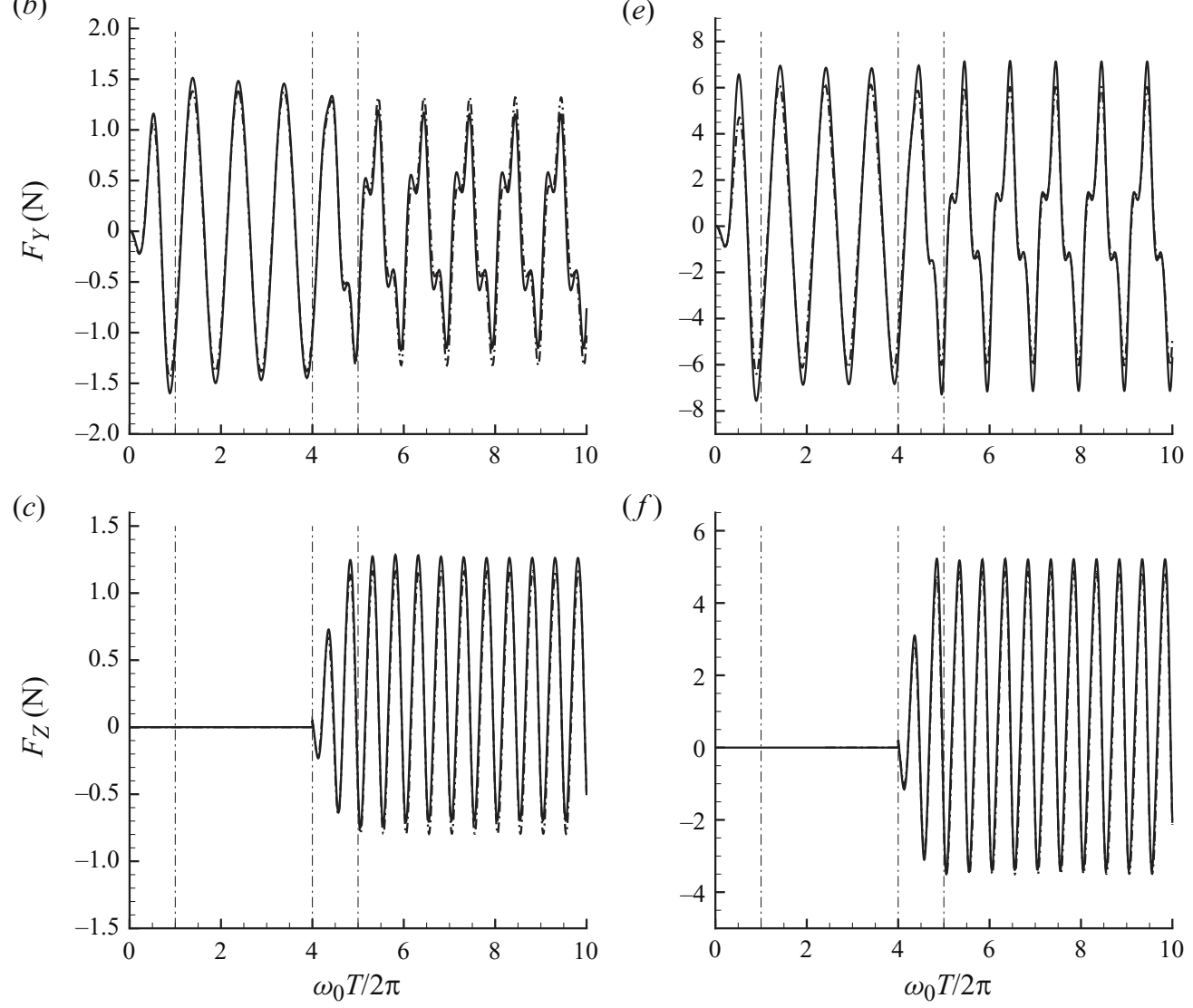

FIGURE 13. Body forces for $(a-c)$ motion 1 and $(d-f)$ motion 2. Lines: - , pressure NS; $-\cdot-\cdot-$, model.

model. Indeed, when correcting the model using the initial value of $F_{X}$ given by the CFD computation, we observed a very good agreement for the EBT-like motion (motion 1), at least before the rotation was activated. This result indicates that when the deformations of the body remain small, the EBT is efficient in predicting the reactive part of the force acting on the body. However, when the unsteady rotation starts, the difference between the two responses becomes significant (as can be seen 

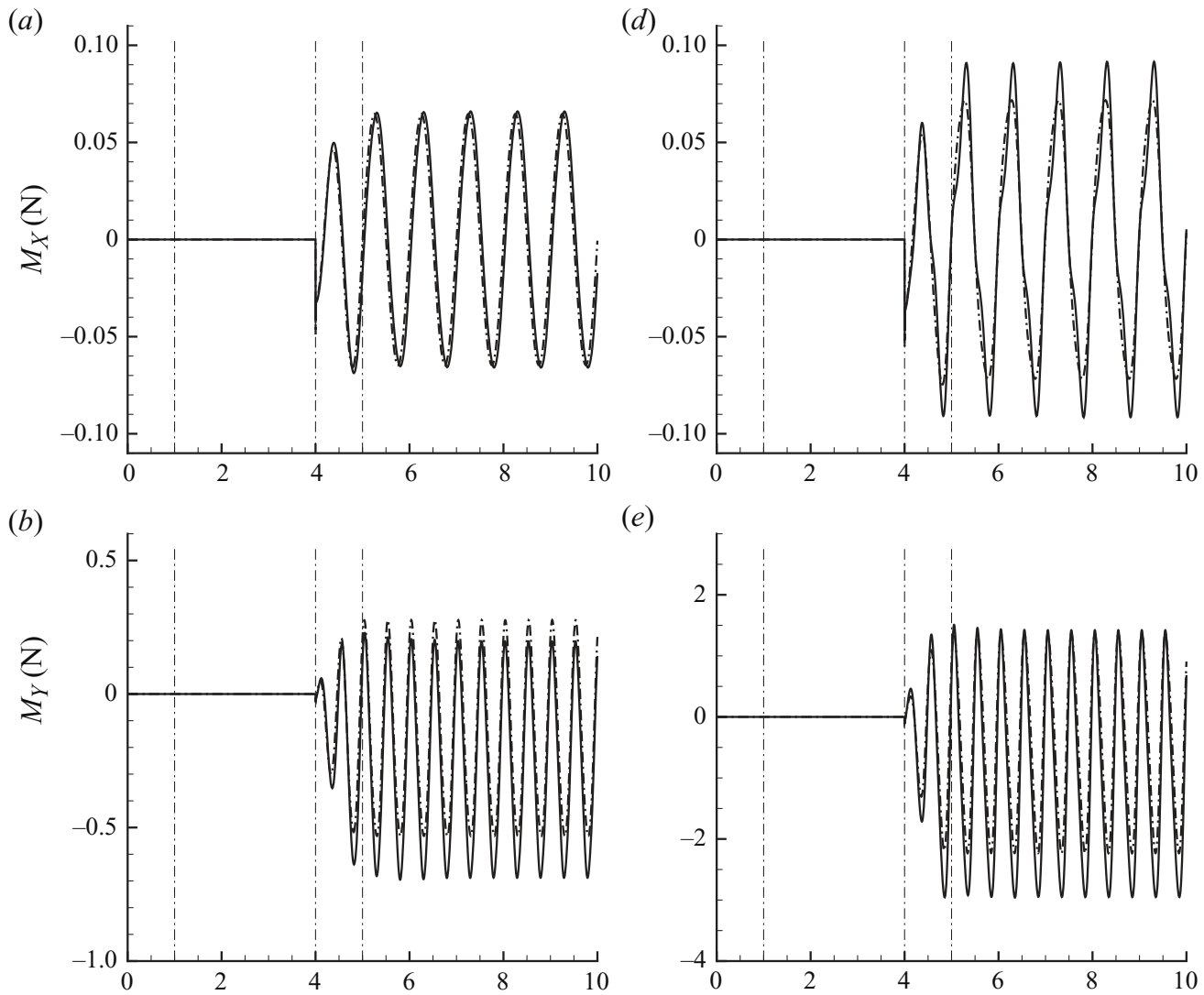

(e)
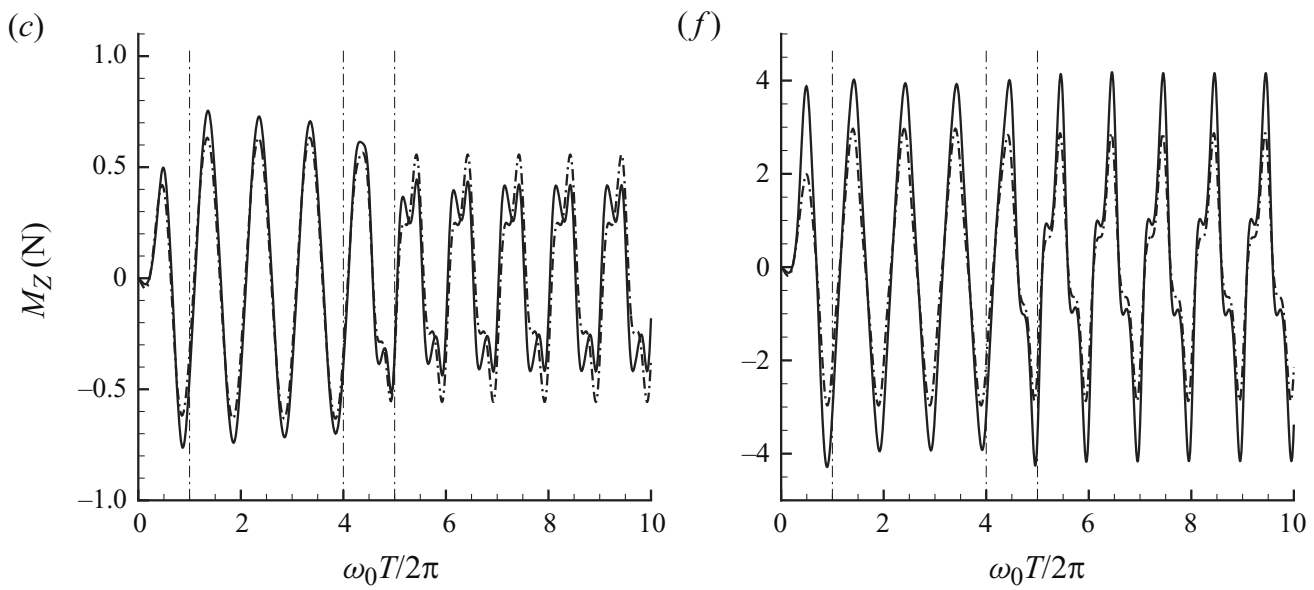

FIGURE 14. Body moments for $(a-c)$ motion 1 and $(d-f)$ motion 2. Lines: — $-\cdot-\cdot-$, model.

in figure $13 a$ after $T=2.5 \mathrm{~s}$ ). As to the responses observed when the second motion is used (motion 2, which involves larger deformation), discrepancies in $F_{X}$ between the numerical results and the model can be observed even before the rotation has started, as can be seen in figure $13(d)$. These results clearly show that the resultant of the pressure forces in the direction of the head is probably the most sensitive to the 
viscous-induced perturbations of flow (i.e. boundary-layer separations) which occur when the deformation of the body becomes significant.

For other components $F_{Y}$ and $F_{Z}$, as well as for the three components of the moment, in contrast to the axial force, the agreement between the model and the numerical simulations is globally very good (even for motion 2 involving large deformations), as can be seen in figures $13(b, c), 13(e, f)$ and 14 . The dynamics are indeed well reproduced by the model, though some discrepancies are sometimes noted, as for example, when the components of the force or the moment reach their maximal values. However, in general, it seems that the added-mass coefficients are only slightly modified by the viscosity effects in such unsteady flows, induced by the two deformations proposed here. Also, note that the forces and moments induced by the viscosity in the Navier-Stokes simulations have not been added in these figures, because they are far weaker than the other ones, except for $F_{X}$ in which the longitudinal viscous forces play a non-negligible role.

In any case, the numerical results suggest that the extended LAEBT appears to be a convenient tool for investigating $3 \mathrm{D}$ transient manoeuvres.

\section{Concluding discussion}

We have provided a 3D extension of the LAEBT, obtaining the expressions of the resultant and moment of pressure exerted on a slender body moving in a perfect fluid, in response to prescribed deformations parametrized by curvature laws. In a comparison with numerical simulations, it has been shown that even though the present model fails to predict accurately the axial force when the imposed curvature becomes significant, it may be used to investigate transient manoeuvres, since all the other components of the hydrodynamic force and moment acting on the body seem to be correctly reproduced by the model.

Note that, for the sake of generality, (3.25) and (3.26) have been obtained in a general case, that is, for arbitrary motions of a slender body (going beyond the case of swimming). However, a reader acquainted with the original LAEBT could naturally wonder if these equations could have been deduced from the balance of the fluid momentum density $\left(\boldsymbol{p}_{f}, \boldsymbol{\sigma}_{f}\right)$ applied to the domain located upstream of the plane $\pi$ (see figure 1) after postulating, as did Lighthill, a stratification of the linear and angular momentum of the fluid along the backbone. This question has been addressed recently by Boyer, Porez \& Leroyer (2009) using a geometrical approach, which gave the following results:

$$
\boldsymbol{f}_{h}=-\frac{\partial}{\partial T} \int_{0}^{l} \boldsymbol{p}_{f} \mathrm{~d} X+\left[V_{X} \boldsymbol{p}_{f}-T_{f} \boldsymbol{t}_{1}\right]_{0}^{l}
$$

and

$$
\boldsymbol{m}_{h}=-\frac{\partial}{\partial T} \int_{0}^{l}\left(\boldsymbol{\sigma}_{f}+\boldsymbol{r} \times \boldsymbol{p}_{f}\right) \mathrm{d} X+\left[V_{X}\left(\boldsymbol{\sigma}_{f}+\boldsymbol{r} \times \boldsymbol{p}_{f}\right)-\boldsymbol{r} \times T_{f} \boldsymbol{t}_{1}\right]_{0}^{l} .
$$

As we can see, all the terms involved in these two equations are included in the more general equations (3.25) and (3.26), but these latter equations also contain additional terms. In order to analyse the contribution of these additional terms during usual swimming of a slender body, let us first specify that in this case, the lateral velocity components of the body, as well as the components of the curvature vector (i.e. $K_{Y}$ and $\left.K_{Z}\right)$, can be generally modelled with functions of the form $f(X \pm c T)$ that correspond to propagating waves, and where $c$ corresponds to the wave speed. These 
wave speeds scale generally like the axial velocity (for instance, it is slightly greater during planar swimming) and this enables us to estimate the order of magnitude of the different terms involving a time derivative, since

$$
\frac{\partial}{\partial T} \sim c \frac{\partial}{\partial X} \sim V_{X} \frac{\partial}{\partial X} .
$$

In addition, as regards the axial angular velocities, by integrating (2.22) with respect to $X$, we obtain

$$
\Omega_{X}=\Omega_{0}+\int_{0}^{X} K_{Z} \Omega_{Y}-K_{Y} \Omega_{Z} \mathrm{~d} X,
$$

where $\Omega_{0}$ (which may be a time-dependent function) stands for the axial angular velocity of the head of the body (i.e. at $X=0$ ). Since the angular velocities $\Omega_{Y}$ and $\Omega_{Z}$ have been shown to be small compared with $\Omega_{X}$ (see (2.25)), then, at leading order, the axial angular velocity of any cross-section is uniform and is given by the axial velocity of the head. During a natural swimming gait, rotation of the head is generally avoided by fish and in any case the condition $\alpha \Omega_{0} \ll V$ is satisfied. If we use this condition, together with (5.3), it turns out that the additional terms existing in (3.25) and (3.26), in comparison with (5.1) and (5.2), are actually negligible in almost all the situations encountered during natural swimming by animals. And, finally, even for 3D manoeuvres, if the rotation of the head has a smooth and slow evolution leading to negligible roll dynamics, the model given by (3.25) and (3.26) has a form similar to the original LAEBT obtained for the 2D planar swimming, except that the linear fluid momentum $\boldsymbol{p}_{f}$ contains an additional component which accounts for the pitch motion of the body.

As regards the parametrization of the body deformation, since the pioneering work of Gray (1933), most of the work on swimming kinematics defines the deformation through an analytical expression of the deflection: the transverse motion of the midline with respect to the axis of movement of the fish. Although this parametrization is easy to relate to the photographs of a fish swimming straight forward, when dealing with more complex gaits (e.g. turns or 3D manoeuvres), it becomes artificially complex, all the more so since the inextensibility constraint is not naturally taken into account (see for example Singh \& Pedley 2008). However, if the shape is parametrized by strain measures (here curvatures), the inextensibility constraint is 'by construction' satisfied since then any shape is deduced from the integration of a strain field compatible with the inextensibility. In fish, this description is quite natural, since the 'curvature is directly related to muscle contractions and strength' (Kern \& Koumoutsakos 2006), and during classical manoeuvres it is often very simple. For instance, when a caranguiform fish is performing a turn manoeuvre, it contorts its body into an arc of circle (as a 'C-curve'), whose radius varies in time (see for instance the experimental results of Anderson 1996), and, in this case, at least at leading order, the curvature, which is uniform along the body, can be written as the simple time law: $K_{Y}=K_{u} h(T)$, where $h(T)$ is a time-dependent function for progressively imposing and subsequently removing the desired curvature.

Finally, it can be said that LAEBT is essentially useful for investigating the leading physical mechanisms involved during transient manoeuvres, where reactive forces and moments are expected to be dominant, and not for investigating self-propelled swimming. In the latter case, if fast simulations must be done, at present it seems that there is no other choice than to add empirical forces and moments to the models, which take into account viscous effect as is proposed for example by Taylor (1952) or, 
more recently, by Galper \& Miloh (2000) or Lopes, Païdoussis \& Semler (2002). Also, another improvement to the LAEBT could be to extend the results of Newman \& $\mathrm{Wu}$ (1973), to account for the effect of the vorticity shed by the fins upstream of the caudal fin.

This work was supported by the European Commission, Information Society and Media, Future and Emerging Technologies (FET) contract 231845.

\section{Appendix. Analytical determination of the potentials $\Phi_{\perp}^{0}$ and $\Phi_{\perp}^{1}$}

In this appendix, solutions $\Phi_{\perp}^{0}$ and $\Phi_{\perp}^{1}$ corresponding to (2.43)-(2.44) and (2.45)(2.46), respectively, are derived. If we look at (2.43) and (2.44), it appears that for each abscissa $X, \Phi_{\perp}^{0}$ corresponds to the $2 \mathrm{D}$ potential in the $(Y, Z)$ plane resulting from the movement (translation and rotation) of an infinite cylinder whose cross-section is $\mathscr{S}_{X}$. In this paper, we consider the cross-sections of the body to be elliptic, and the usual way to derive the $2 \mathrm{D}$ potential in such a case is to use a conformal transformation that links the elliptic section to a circular section. However, in the present investigation, and for later convenience, we prefer to use elliptic coordinates $(u, v)$, where $u \geqslant 0$ and $0 \leqslant v \leqslant 2 \pi$, since such coordinates are also used to obtain the expression of $\Phi_{\perp}^{1}$, where conformal transformation can no longer be used ( $\Phi_{\perp}^{1}$ is not a harmonic function). In this investigation, we are concerned with elliptic cross-sections whose major and minor semi-axes are $\alpha$ and $\beta$, respectively. Furthermore, we have chosen to position both foci of the elliptic cross-section of the body on the $Y$-axis, the distance between the foci being $2 a$, where $a$ is linked to the major and minor axes by the relation

$$
a=\sqrt{\alpha^{2}-\beta^{2}} .
$$

Elliptic coordinates $(u, v)$, where $u \geqslant 0$ and $0 \leqslant v \leqslant 2 \pi$, are linked to the classical Cartesian coordinates by the following relations:

$$
Y=a \cosh (u) \cos (v) \quad \text { and } \quad Z=a \sinh (u) \sin (v),
$$

and the boundary of an elliptic cross-section simply reads $u=u_{X}$, where the value of $u_{X}$ is linked to $\alpha$ and $\beta$ by the following relations:

$$
\alpha=a \cosh \left(u_{X}\right) \quad \text { and } \quad \beta=a \sinh \left(u_{X}\right) \quad \text { or, reciprocally } u_{X}=\frac{1}{2} \ln ((\alpha+\beta) /(\alpha-\beta)) \text {, }
$$

so that here $u_{X}$ depends on $X$ since the size of the cross-section of the body may change along the body.

Elliptic coordinates are orthogonal and the tangential unit vectors, namely $\boldsymbol{e}_{u}$ and $\boldsymbol{e}_{v}$, are given by

where

$$
\begin{gathered}
\boldsymbol{e}_{u}=\frac{a}{J(u, v)^{1 / 2}}\left(\sinh (u) \cos (v) \boldsymbol{t}_{2}+\cosh (u) \sin (v) \boldsymbol{t}_{3}\right), \\
\boldsymbol{e}_{v}=\frac{a}{J(u, v)^{1 / 2}}\left(-\cosh (u) \sin (v) \boldsymbol{t}_{2}+\sinh (u) \cos (v) \boldsymbol{t}_{3}\right),
\end{gathered}
$$

$$
J=a^{2} \sinh (u)^{2}+a^{2} \sin (v)^{2}
$$

is the Jacobian determinant of the elliptic coordinates. We also specify that, for any function $f(u, v)$, the $2 \mathrm{D}$ gradient and the Laplacian read

$$
\nabla f=\frac{1}{J^{1 / 2}}\left(\frac{\partial f}{\partial u} \boldsymbol{e}_{u}+\frac{\partial f}{\partial v} \boldsymbol{e}_{v}\right) \quad \text { and } \quad \Delta f=\frac{1}{J}\left(\frac{\partial^{2} f}{\partial u^{2}}+\frac{\partial^{2} f}{\partial v^{2}}\right) .
$$




\section{A.1. Solution $\Phi_{\perp}^{0}$}

In terms of elliptic coordinates and since in this problem $N_{Y} \boldsymbol{t}_{2}+N_{Z} \boldsymbol{t}_{3}=\boldsymbol{e}_{u}+O\left(\varepsilon^{2}\right)$, (2.43) and (2.44) governing $\Phi_{\perp}^{0}$ read

$$
\begin{gathered}
\frac{\partial^{2} \Phi_{\perp}^{0}}{\partial u^{2}}+\frac{\partial^{2} \Phi_{\perp}^{0}}{\partial v^{2}}=0, \\
\left.\frac{\partial \Phi_{\perp}^{0}}{\partial u}\right|_{u_{X}}=\beta V_{Y} \cos v+\alpha V_{Z} \sin (v)+\frac{\Omega_{X} a^{2}}{2} \sin (2 v),
\end{gathered}
$$

and, necessarily, we also have

$$
\nabla \Phi_{\perp}^{0} \rightarrow \mathbf{0}, \quad|u| \rightarrow \infty .
$$

Since the potential $\Phi_{\perp}^{0}$ must be $2 \pi$-periodic with respect to $v$, it is convenient to seek it by using a Fourier series

$$
\Phi_{\perp}^{0}=\sum_{-\infty}^{\infty} F_{n}(u) \exp (\mathrm{i} n v)
$$

where ' $i$ ' is the imaginary number. Then, if we introduce (A 11) into (A 8) and exploit the orthogonality properties of the complex exponential, it is quite simple to show that, in terms of elliptic coordinates, the very general solution of the $2 \mathrm{D}$ Laplacian is

$$
\begin{aligned}
\Phi_{\perp}^{0}=A_{0}+B_{0} u+\sum_{n=1}^{\infty}\left(A_{n} \cos (n v)+B_{n} \sin (n v)\right) & \exp (-n u)+\left(C_{n} \cos (n v)\right. \\
& \left.+D_{n} \sin (n v)\right) \exp (n u),
\end{aligned}
$$

where coefficients $A_{n}, B_{n}, C_{n}$ and $D_{n}$ are constants that must be determined by the boundary conditions. Here, (A 10) imposes taking only decreasing harmonics into account so that $C_{n}=D_{n}=0$, and according to (A 9), we obtain

$\Phi_{\perp}^{0}=-\frac{a^{2} \Omega_{X}}{4} \sin (2 v) \exp \left(2 u_{X}-2 u\right)-\beta V_{Y} \cos (v) \exp \left(u_{X}-u\right)-\alpha V_{Z} \sin (v) \exp \left(u_{X}-u\right)$,

where we have neglected a constant. Note that (A 13) is obviously not an original result and this solution can be found in many classical books on fluid mechanics (see for instance Milne-Thomson 1968, pp. 258-259). However, what is interesting here is how this solution has been derived, since the same approach will be used to determine $\Phi_{\perp}^{1}$, where the expression of $\Phi_{\perp}^{0}$ is also involved.

\section{A.2. Solution $\Phi_{\perp}^{1}$}

In terms of elliptic coordinates, partial derivatives of the right-hand side of (2.45) read

$$
\frac{\partial}{\partial Y}=\frac{a}{J}\left(\sinh (u) \cos (v) \frac{\partial}{\partial u}-\cosh (u) \sin (v) \frac{\partial}{\partial v}\right)
$$

and

$$
\frac{\partial}{\partial Z}=\frac{a}{J}\left(\cosh (u) \sin (v) \frac{\partial}{\partial u}+\sinh (u) \cos (v) \frac{\partial}{\partial v}\right),
$$


where $J$ is given by (A 6). According to (A 13), it may be shown, after some algebra, that $(2.45)$ can be written as follows:

$$
\begin{aligned}
\frac{\partial^{2} \Phi_{\perp}^{1}}{\partial u^{2}}+\frac{\partial^{2} \Phi_{\perp}^{1}}{\partial v^{2}}= & \left(\mathscr{C}_{-3} \exp (-3 \mathrm{i} v)+\mathscr{C}_{3} \exp (3 \mathrm{i} v)\right) \exp (-u)+\left(\mathscr{C}_{-2} \exp (-2 \mathrm{i} v)\right. \\
& \left.+\mathscr{C}_{2} \exp (2 \mathrm{i} v)\right)+\left(\mathscr{C}_{-1} \exp (-\mathrm{i} v)+\mathscr{C}_{1} \exp (\mathrm{i} v)\right) \exp (-3 u) \\
& +\mathscr{C}_{0} \exp (-2 u)
\end{aligned}
$$

where

$$
\begin{gathered}
\mathscr{C}_{-3}=\frac{a^{3} \exp \left(2 u_{X}\right)}{8} \Omega_{X}\left(K_{Y}+\mathrm{i} K_{Z}\right), \quad \mathscr{C}_{3}=\text { c.c. }\left(\mathscr{C}_{-3}\right), \\
\mathscr{C}_{-2}=\frac{a \exp \left(u_{X}\right)}{4}\left(\left(K_{Z} V_{Y} \beta+K_{Y} V_{Z} \alpha\right)+\mathrm{i}\left(K_{Z} V_{Z} \alpha-K_{Y} V_{Y} \beta\right)\right), \quad \mathscr{C}_{2}=\text { c.c. }\left(\mathscr{C}_{-2}\right), \\
\mathscr{C}_{-1}=-\mathscr{A}_{-3}, \quad \mathscr{C}_{1}=\text { c.c. }\left(\mathscr{C}_{-1}\right),
\end{gathered}
$$

and

$$
\mathscr{C}_{0}=-\frac{a \exp \left(u_{X}\right)}{2}\left(K_{Z} V_{Y} \beta+K_{Y} V_{Z} \alpha\right)
$$

(Here c.c. stands for complex conjugate.) Also, the boundary conditions are

$$
\left.\frac{\partial \Phi_{\perp}^{1}}{\partial u}\right|_{u_{X}}=0 \quad \text { and } \quad \nabla \Phi_{\perp}^{1} \rightarrow 0 \quad|u| \rightarrow \infty .
$$

Once again, since $\Phi_{\perp}^{1}$ is also $2 \pi$-periodic, the solution can be sought in the form

$$
\Phi_{\perp}^{1}=\sum_{-\infty}^{\infty} G_{n}(u) \exp (\mathrm{i} n v) .
$$

After injecting this sum into (A 16), and after using complex exponential orthogonal properties, we obtain a set of ordinary differential equations of the form

$$
\frac{\mathrm{d}^{2} G_{n}}{\mathrm{~d} u^{2}}-n^{2} G_{n}=\mathscr{C}_{n} \exp (-m u), \quad n \in \mathbb{Z},
$$

and $m$ is equal to $-1,-2$ or -3 . General solutions of these ordinary differential equations read

or

$$
G_{0}(u)=A_{0}+B_{0} u+\frac{\mathscr{C}_{0}}{m^{2}} \exp (-m u), \quad \text { if } n=0
$$

$$
G_{n}=A_{n} \exp (n u)+B_{n} \exp (-n u)-\frac{\mathscr{C}_{n}}{n^{2}-m^{2}} \exp (-m u), \quad \text { if } n \neq 0,
$$

where $A_{n}$ and $B_{n}$ are constants which must be determined by the boundary conditions. According to (A 21), we obtain the solution

$$
\begin{aligned}
\Phi_{\perp}^{1}= & -\frac{a \exp \left(-u_{X}\right)}{4}\left(K_{Z} \beta V_{Y}+K_{Y} \alpha V_{Z}\right)\left(u+\frac{1}{2} \exp \left(2 u_{X}-2 u\right)\right) \\
& +\frac{a^{3} \Omega_{X}}{32} \exp \left(-u_{X}\right)\left(3 \exp \left(u_{X}-u\right)-\exp \left(3 u_{X}-3 u\right)\right)\left(K_{Y} \cos (v)+K_{Z} \sin (v)\right) \\
& -\frac{a \exp \left(u_{X}\right)}{8}\left(\left(K_{Z} \beta V_{Y}+K_{Y} \alpha V_{Z}\right) \cos (2 v)+\left(K_{Z} \alpha V_{Z}-K_{Y} \beta V_{Y}\right) \sin (2 v)\right) \\
& +\frac{a^{3} \Omega_{X}}{32} \exp \left(u_{X}\right)\left(\frac{1}{3} \exp \left(3 u_{X}-3 u\right)-\exp \left(u_{X}-u\right)\right)\left(K_{Y} \cos (3 v)+K_{Z} \sin (3 v)\right) .
\end{aligned}
$$




\section{A.3. Determination of $\Phi_{\|}$}

In order to determine the part $\Phi_{\|}$of the velocity potential, we first need to determine analytically the expression of the component $N_{X}$ of the outward normal of the body surface lying between the two sections $\mathscr{S}_{X}$ and $\mathscr{S}_{X+\mathrm{d} X}$. To determine $N_{X}$, we first need to determine the expression of the spatial increments $\mathrm{d} l$ and $\mathrm{d} s$ (see figure 8). Here, we are concerned with the frontal surface (along $\boldsymbol{t}_{1}$ ) lying between two elliptic cross-sections (whose major and minor axes are, respectively, $\alpha(X)$ and $\alpha(X+\mathrm{d} X)$ and $\beta(X)$ and $\beta(X+\mathrm{d} X))$. Then, the implicit equation of the contour of $\mathscr{S}_{X+\mathrm{d} X}$ reads

$$
\frac{Y^{2}}{\alpha(X+\mathrm{d} X)^{2}}+\frac{Z^{2}}{\beta(X+\mathrm{d} X)^{2}}=1 .
$$

Furthermore, in terms of elliptic coordinates, we also have

$$
Y=a(X) \cosh \left(u_{X}+\delta u\right) \cos (v) \quad \text { and } \quad Z=a(X) \sinh \left(u_{X}+\delta u\right) \sin (v),
$$

where $\delta u$ is the increment of the dimensionless elliptic coordinates $u_{X}$ that must be added to reach the contour of $\mathscr{S}_{X+\mathrm{d} X}$ from the contour of $\mathscr{S}_{X}$. If we introduce these expressions of $Y$ and $Z$ into (A 27), we can show, by using a classical series expansion (with respect to $\mathrm{d} X$ ), that this increment is given by

$$
\delta u=\frac{\frac{\partial \alpha}{\partial X} \beta \cos (v)^{2}+\alpha \frac{\partial \beta}{\partial X} \sin (v)^{2}}{J\left(u_{X}, v\right)} \mathrm{d} X+O\left(\mathrm{~d} X^{2}\right),
$$

where $J\left(u_{X}, v\right)$ is the Jacobian determinant corresponding to elliptic coordinates, evaluated at the elliptic coordinates $u_{X}$ :

$$
J\left(u_{X}, v\right)=\beta(X)^{2} \cos (v)^{2}+\alpha(X)^{2} \sin (v)^{2} .
$$

If we return to the spatial increments $\mathrm{d} l$ and $\mathrm{d} s$, we have

$$
\mathrm{d} l=J\left(u_{X}, v\right)^{1 / 2} \delta u \quad \text { and } \quad \mathrm{d} s=J\left(u_{X}, v\right)^{1 / 2} \mathrm{~d} v .
$$

It is straightforward to show that

$$
N_{X}=-\frac{\mathrm{d} l}{\sqrt{\mathrm{d} X^{2}+\mathrm{d} l^{2}}}
$$

which leads to the result

$$
N_{X}=-\frac{\beta \cos (v)^{2} \frac{\partial \alpha}{\partial X}+\alpha \sin (v)^{2} \frac{\partial \beta}{\partial X}}{J\left(u_{X}, v\right)^{1 / 2}}+O\left(\varepsilon^{2}\right) .
$$

In these conditions, $\Phi_{\|}$can be obtained by following the same approach (for deriving $\left.\Phi_{\perp}^{0}\right)$ to obtain

$$
\Phi_{\|}=-\frac{V_{X}}{2}\left(\left(\frac{\partial \alpha}{\partial X} \beta+\frac{\partial \beta}{\partial X} \alpha\right) u-\frac{1}{2}\left(\frac{\partial \alpha}{\partial X} \beta-\frac{\partial \beta}{\partial X} \alpha\right) \cos (2 v) \exp \left(2 u_{X}-2 u\right)\right) .
$$

\section{REFERENCES}

ANDERSON, J. 1996 Vorticity control for efficient propulsion. PhD thesis, Massachussets Institute of

Technology and the Woods Hole Ocean Engineering.

Batchelor, G. K. 1967 An Introduction to Fluid Dynamics. Cambridge University Press. 
BlaKe, R. W. 2004 Fish functional design and swimming performance. J. Fish Biol. 65 (5), 1193-1222.

Boyer, F., Porez, M. \& Leroyer, A. 2009 Poincaré-Cosserat equations for the Lighthill threedimensional large-amplitude elongated body theory: application to robotics. J. Nonlinear Sci. 20 (1), 47-79.

Boyer, F., Porez, M., Leroyer, A. \& Visonneau, M. 2008 Macro-continuous computed torque algorithm for a three-dimensional eel-like robot. IEEE Trans. Robot. 22, 975-991.

Childress, S. 1981 Mechanics of Swimming and Flying. Cambridge University Press.

Colgate, J. E. \& LYNCH, K. M. 2004 Mechanics and control of swimming: a review. IEEE J. Ocean. Engng 29, 660-673.

ELDREDGE, J. D. 2008 Dynamically coupled fluid-body interactions in vorticity-based numerical simulations. J. Comput. Phys. 227, 9170-9194.

Galper, A. \& Miloh, T. 1995 Dynamic equations of motion for a rigid or deformable body in an arbitrary non-uniform potential flow field. J. Fluid Mech. 295, 91-120.

Galper, A. R. \& Miloh, T. 1999 Curved slender structures in non-uniform flows. J. Fluid Mech. $385,21-40$.

GalPer, A. R. \& Miloh, T. 2000 Hydroelasticity of the Kirchhoff rod: buckling phenomena. $J$. Fluids Struct. 14, 1089-1100.

GraY, J. 1933 Studies in animal locomotion. I. The movement of fish with special reference to eel. J. Expl. Biol. 10, 88-104.

Kanso, E. 2009 Swimming due to transverse shape deformations. J. Fluid Mech. 631, 127-148.

Kanso, E., Marsden, J. E., Rowley, C. W. \& Melli-Huber, J. B. 2005 Locomotion of articulated bodies in a perfect fluid. J. Nonlinear Sci. 15 (4), 255-289.

Kern, S. \& Koumoutsakos, P. 2006 Simulation of optimized anguilliform swimming. J. Expl. Biol. 209, 4841-4857.

LERoYer, A. \& VisonNEAU, M. 2005 Numerical methods for RANSE simulations of a self-propelled fish-like body. J. Fluids Struct. 20, 763-775.

Lighthill, M. J. 1960 Note on the swimming of slender fish. J. Fluid Mech. 9 (2), 305-317.

Lighthill, M. J. 1970 Aquatic animal propulsion of high hydromechanical efficiency. J. Fluid Mech. 44, 265-301.

Lighthill, M. J. 1975 Mathematical Biofluiddynamics. Regional Conference Series in Applied Mathematics, vol. 17. SIAM.

Lopes, J.-L., Païdoussis, M. P. \& Semler, C. 2002 Linear and nonlinear dynamics of cantilevered cylinders in axial flow. Part 2. The equations of motion. J. Fluids Struct. 16 (6), 715-737.

Milne-Thomson, L. M. 1968 Theoretical Hydrodynamics. Dover.

Muller, U. K., Stamhuis, E. J. \& Videler, J. J. 2000 Hydrodynamics of unsteady fish swimming and the effects of body size: comparing the flow fields of fish larvae and adults. J. Expl. Biol. 203 (2), 193-206.

MunNIER, A. 2009 Locomotion of deformable bodies in an ideal fluid: Newtonian versus Lagrangian formalisms. J. Nonlinear Sci. 19 (6), 665-715.

Newman, J. N. 1973 The force on a slender fish-like body. J. Fluid Mech. 58 (4), 689-702.

Newman, J. N. \& Wu, T. Y. 1973 A generalized slender-body theory for fish-like forms. J. Fluid Mech. 57 (4), 673-693.

ReISSNER, E. 1973 On a one-dimensional large displacement finite-strain theory. Stud. Appl. Maths 52, 87-95.

Sfakiotakis, M., Lane, D. M. \& Davies, J. B. C. 1999 Review of fish swimming modes for aquatic locomotion. IEEE J. Ocean. Engng 24, 237-252.

Simo, J. C. \& VU-LocQ, L. 1988 On the dynamics in space of rods undergoing large motions: a geometrically exact approach. Intl J. Numer. Meth. Engng 66, 125-161.

Singh, K. \& Pedley, T. J. 2008 The hydrodynamics of flexible body manoeuvres in swimming fish. Physica D: Nonlinear Phenom. 237 (14-17), 2234-2239.

Sparenberg, J. A. 2002 Survey of the mathematical theory of fish locomotion. J. Engng Maths 44, 395-448.

TAYLOR, G. 1952 Analysis of the swimming of long and narrow animals. Proc. R. Soc. Lond. A 214, $158-183$.

Triantafyllou, M. S., Triantafyllou, G. S. \& Yue, D. K. P. 2000 Hydrodynamics of fishlike swimming. Annu. Rev. Fluid Mech. 32, 33-53. 
Tytell, E. D. 2004 The hydrodynamics of eel swimming. II. Effect of swimming speed. J. Expl. Biol. 207, 3265-3279.

Weirs, D. 1972 A hydrodynamical analysis of fish turning manoeuvres. Proc. R. Soc. Lond. B 182, 59-72.

WeIHS, D. 1973 The mechanism of rapid starting of slender fish. Biorheology 10, 343-350.

WolfGang, M. J. 1999 Hydrodynamics of flexible-body swimming motions. PhD thesis, Massachussets Institute of Technology.

Wu, T. Y. 1961 Swimming of the waving plate. J. Fluid Mech. 10, 321-344.

Zhu, Q., Wolfgang, M. J., Yue, D. K. P. \& Triantafyllou, M. S. 2002 Three-dimensional flow structures and vorticity control in fish-like swimming. J. Fluid Mech. 468, 1-28. 